\title{
DYNAMIC RESPONSE OF A PAIR OF WALLS RETAINING A VISCOELASTIC SOLID
}

A.S. Veletsos, V.H. Parikh, A.H. Younan and K. Bandyopadhyay

January 1995

Prepared for

OFFICE OF ENVIRONMENTAL RESTORATION AND WASTE MANAGEMENT DEPARTMENT OF ENERGY, WASHINGTON, D.C. 


\section{DISCLAIMER}

This report was prepared as an account of work sponsored by an agency of the United States Government. Neither the United States Government nor any agency thereof nor any of their employees, nor any of their contractors, subcontractors, or their employees, makes any warranty, express or implied, or assumes any legal liability or responsibility for the accuracy, completeness, or usefulness of any information, apparatus, product, or process disclosed, or represents that its use would not infringe privately owned rights. Reference herein to any specific commercial product, process, or service by trade name, trademark, manufacturer, or otherwise, does not necessarily constitute or imply its endorsement, recommendation, or favoring by the United States Government or any agency, contractor or subcontractor thereof. The views and opinions of authors expressed herein do not necessarily state or reflect those of the United States Govemment or any agency, contractor or subcontractor thereof.

Printed in the United States of America

Available from

National Technical Information Service

U.S. Department of Commerce

5285 Port Royal Road

Springfield, VA 22161

NTIS price codes:

Printed Copy: A04; Microfiche Copy: A01 


\section{DISCLAIMER}

Portions of this document may be illegible in electronic image products. Images are produced from the best available original document. 


\begin{abstract}
Making use of a previously reported, simple, approximate method of analysis, a critical evaluation is made of the dynamic pressures and forces induced by horizontal ground shaking on a pair of infinitely long, parallel walls retaining a uniform viscoelastic solid. The walls are presumed to be rigid but elastically constrained against rotation at their base. The effects of both harmonic and earthquake-induced excitations are examined. The accuracy of the method is assessed by comparing its predictions for the special case of fixed-based walls with those obtained by an exact method, and comprehensive numerical data are presented which elucidate the underlying response mechanisms, and the effects and relative importance of the parameters involved. The parameters examined include the characteristics of the ground motion, the ratio of the distance between walls to the height of the contained material, and the flexibility of the rotational wall constraints. In addition to valuable insights into the responses of the systems investigated, the results presented provide a convenient framework for the analysis of more complex systems as well.
\end{abstract}



TABLE OF CONTENTS

Section

Page

ABSTRACT

iii

TABLE OF CONTENTS

$\mathrm{v}$

LIST OF FIGURES

vii

LIST OF TABLES

ix

EXECUTIVE SUMMARY

xi

ACKNOWLEDGMENT

xiii

1 INTRODUCTION

$1-1$

2 SYSTEM AND METHOD OF ANALYSIS

2-1

2.1 System Considered

2.2 Governing Equations and Assumptions

2-1

2.3 Solution for Harmonic Response

$2-3$

2.4 Foundation Moment

2-6

2.5 Computation of Transient Response

2-7

3 RESPONSE OF SYSTEMS WITH FIXED-BASED WALLS

3-1

3.1 Static Effects

3-1

3.2 Results for Harmonic Response

3-2

3.3 Results for Transient Response

3-4

4 RESPONSE OF SYSTEMS WITH ROTATIONALLY CONSTRAINED WALLS

4-1

5.1 Static Effects

4-1

5.2 Fundamental Natural Period of System

4-1

5.3 Resutls for Dynamic Response

4-2

5.4 Contribution of Higher Modes

5 CONCLUSIONS

6 REFERENCES

7 NOTATION 


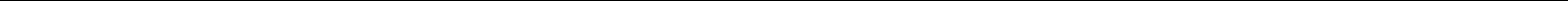




\section{LIST OF FIGURES}

Figure $\quad$ Page

2.1 System Considered. $\quad 2-8$

3.1 Static values of base shear and base moment per unit of length of each wall for systems with fixed-based walls; $\mathrm{m}_{\mathrm{w}}=0, v=0.3$.

3.2 Distributions of static wall pressures for systems with fixed-based walls; $\mathrm{m}_{\mathrm{w}}=0, \mathrm{v}=0.3$.

3.3 Distributions of static base pressures for systems with fixed-based walls; $\mathrm{m}_{\mathrm{w}}=0, \mathrm{v}=0.3$.

3.4 Amplitude of base shear per unit of length of each wall for harmonically excited systems with fixed-based walls; $\mathrm{m}_{\mathrm{w}}=0, v=0.3, \delta=0.1$.

3.5 Absolute maximum amplification factor for base shear of systems with fixed-based walls subjected to harmonic and transient excitations; $m_{w}=0, v=0.3, \delta=0.1$.

3.6 Comparison of frequency response curves for base shear in wall of systems computed considering many and a single vertical mode of vibration; $\mathrm{m}_{\mathrm{w}}=0$, $v=0.3, \delta=0.1$.

3.7 Amplification factors for maximum base wall-shear computed for El Centro record considering many and a single vertical mode of vibration; $\mathrm{m}_{\mathrm{w}}=0, v=0.3$, $\delta=0.1$.

4.1 Effects of rotational flexibility of walls on static values of: (a) base shear per unit of wall length; and (b) effective height; $\mathrm{m}_{\mathrm{w}}=0, v=0.3$.

4.2 Effect of rotational flexibility of wall on fundamental natural period of vibration of systems with different aspect ratios; $\mathrm{m}_{\mathrm{w}}=0, v=0.3$.

4.3 Maximum base shear per unit of length of each wall for systems with elastically constrained walls subjected to El Centro record; $\mathrm{m}_{\mathrm{w}}=0, v=0.3, \delta=0.1$, $\delta_{\mathrm{w}}=0.04$. 
4.4 Absolute maximum value of base shear per unit of length of each wall for systems with elastically constrained walls subjected to the El Centro record; $\mathrm{m}_{\mathrm{w}}=0$, $v=0.3, \delta=0.1, \delta_{\mathrm{w}}=0.04$.

4.5 Absolute maximum value of base shear per unit of length of each wall for harmonically excited systems with elastically constrained walls; $\mathrm{m}_{\mathrm{w}}=0, \mathrm{v}=0.3, \delta=0.1$, $\delta_{\mathrm{w}}=0.04$.

4.6 Absolute maximum amplification factor for base wall-shear for systems with elastically constrained walls subjected to: (a) the El Centro record, and (b) harmonic base motions; $\mathrm{m}_{\mathrm{w}}=0, v=0.3, \delta=0.1, \delta_{\mathrm{w}}=0.04$.

4.7 Comparison of maximum base wall-shear for systems with elastically constrained walls computed by considering many and a single vertical mode of vibration;

El Centro input, $\mathrm{m}_{\mathrm{w}}=0, v=0.3, \delta=0.1, \delta_{\mathrm{w}}=0.04$.

4.8 Effective height $h$ of systems with elastically constrained walls subjected to the El Centro record; $\mathrm{m}_{\mathrm{w}}=0, v=0.3, \delta=0.1, \delta_{\mathrm{w}}=0.04$. 


\section{LIST OF TABLES}

Table

Page

3.1 Static values of base shear, $\left(Q_{b}\right)_{s t}$, and of base moment, $\left(M_{b}\right)_{s t}$, for systems with fixed-based walls; $\mathrm{m}_{\mathrm{w}}=0$ and $\mathrm{v}=0.3$.

4.1 Static values of base shear $\left(Q_{b}\right)_{s t}$, of effective height $h$, and of fundamental natural period $T_{11}$ for systems with elastically constrained walls;

$v=0.3$ and $m_{w}=0$. 


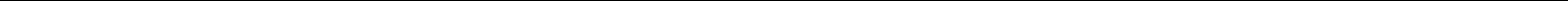




\section{EXECUTIVE SUMMARY}

The study reported herein is motivated by the need for improved understanding of the response to earthquakes of tanks in nuclear facilities storing high-level radioactive wastes. The study complements those reported previously in Brookhaven National Laboratory reports 52378, 52417 and 52420. Whereas the previous studies dealt with the response of tanks containing homogeneous, layered, or continuously inhomogeneous liquids, in the present study the tank content is modeled as a homogeneous viscoelastic solid.

The specific system examined consists of a rectangular strip of viscoelastic material which is free at its upper surface, is fully bonded to a rigid base, and is retained along its long vertical boundaries by rigid walls that are elastically constrained against rotation at their base. The bases of both the medium and the walls are presumed to be excited by a space-invariant, uniform horizontal motion.

For the special case of fixed-based walls, the response of this system was analyzed previously by a precise method. This method requires the evaluation of double series, is computationlly quite intensive, and must be implemented with extreme care, as it may lead to numerical instabilities, particularly when the distance between walls is large compared to the thickness of the retained material. Several simpler, approximate methods have been proposed for the limiting case of a semiinfinite layer retained by a single wall, and it is one of the objectives of the present study to apply the most appropriate of these methods to the two-wall system considered here. Additional objectives are: (1) to assess the accuracy of the proposed method by comparing its predictions with those obtained by the precise method; (2) to further extend the solution to a viscoelastic medium retained by walls that are rigid but elastically constrained against rotation at their base; and (3) to provide numerical data that elucidate the underlying response mechanisms and the effects and relative importance of the numerous parameters involved. The rotationally constrained system is examined as a first step toward a more refined study of the effects of wall flexibility on the response of tanks containing a viscoelastic solid.

In addition to the characteristics of the ground motion and the physical properties of the retained material, the problem parameters considered include the aspect ratio of the system and the rotational flexibility of the wall constraints. Both harmonic and transient ground motions are examined. The response quantities studied include the maximum values of the dynamic wall pressures and of the associated base shear and base moment. 
The principal conclusions of the study may be summarized as follows:

1. Almost independently of the characteristics of the ground motion, the magnitudes of the wall pressures and of the associated wall forces induced by ground shaking are highly dependent on the ratio $\mathrm{L} / \mathrm{H}$ of the distance between walls to the height of the retained material. For very slender systems with small values of $\mathrm{L} / \mathrm{H}$, the inertia forces for all the contained material are effectively transmitted to the walls by horizontal extensional action, and practically the entire mass of the medium may be considered to be effective. With increasing $\mathrm{L} / \mathrm{H}$, a progressively larger portion of the inertia forces gets transferred by horizontal shearing action to the base, and the portion of the retained mass that contributes to the wall forces is reduced. Additionally, an increase in $\mathrm{L} / \mathrm{H}$ increases the damping capacity of the system and reduces the dynamic amplification factors for the wall forces.

2. Provided the aspect ratio of the system $\mathrm{L} / \mathrm{H}$ is not very small, the effect of wall flexibility is to reduce the horizontal extensional stiffness of the contained material relative to its shearing stiffness, and this reduction, in turn, reduces the magnitudes of the resulting wall forces. This result is in contradiction to the well established behavior of liquid-containing tanks, for which the effect of wall flexibility is to increase rather than decrease the dominating, impulsive components of response. However, as L/H tends to zero, the medium's ability to transfer forces vertically by horizontal shearing action vanishes, the medium effectively acts as a liquid, and, in conformance with the known response of liquid containing tanks, the flexibility of the wall increases the dynamic effects. For the range of parameters likely to be encountered in practice, the dynamic wall forces for tanks containing a viscoelastic solid are likely to decrease rather than increase with increasing wall flexibility.

3. While the low-frequency, static values of the wall forces decrease with decreasing distance between walls, the maximum dynamic forces first increase and then decrease. The increased dynamic effects are due to the reduced damping capacity of the finite-sized, narrower systems.

4. For systems with fixed-based rigid walls, an excellent approximation to the response may be obtained by considering the contribution of only the fundamental vertical mode of vibration. By contrast, for systems with elastically constrained walls, the contributions of the second and higher vertical modes may also be important. The contributions of all horizontal modes of vibration are provided for directly in the method of analysis employed.

5. Because of the assumption of vanishing vertical normal stresses underlying the simplified method of analysis, the component of the foundation moment contributed by the dynamic pressures acting on the tank base cannot be evaluated. However, the total foundation moment may be determined directly from the inertia forces of the retained medium, and, if desired, the component contributed by the base pressures may be determined by substracting from the total moment the moments at the base of the walls. 


\section{ACKNOWLEDGMENT}

This study was carried out at Rice University in cooperation with Brookhaven National Laboratory (BNL). The authors are grateful to the Department of Energy Program Directors John Tseng, James Antizzo and Dinesh Gupta for supporting the study, and to Dr. Morris Reich of BNL for his understanding project management. Comments received from colleagues of BNL's Tank Seismic Experts Panel are also acknowledged with thanks. 



\section{SECTION 1 \\ INTRODUCTION}

As far as can be determined, the most comprehensive and accurate study of the dynamic soil pressures induced by ground shaking on vertical walls of embedded structures is the one presented by Wood [1, 2]. The system examined in this study is a rectangular strip of soil that is supported on a nondeformable base and is retained along its long faces by fixed-based, rigid vertical walls. The system is presumed to be excited by a space-invariant horizontal base motion, and the retained material is presumed to respond in its linearly elastic range. This system also models a laterally excited, long rectangular, rigid tank containing an elastic solid. The dynamic response of the latter system is of current interest in connection with the evaluation of the seismic performance of tanks in nuclear facilities storing radioactive wastes, which in some cases may more appropriately be modeled as deformable elastic solids rather than as incompressible liquids.

For the conditions considered, Wood's solution is exact and applicable to arbitrary ratios of the distance between walls to height of the retained material. However, the solution requires the evaluation of double series, is computationally quite intensive, and must be computed with extreme care, as it may lead to numerical instabilities, particularly when the distance between walls is large compared to the thickness of the retained material. Several simpler, approximate methods have been proposed for the limiting case of a semi-infinite layer retained by a single wall $[3,4,5,6]$, and it is one of the objectives of the present study to apply the most appropriate of these methods to the two-wall system considered here. Additional objectives are: (1) to assess the accuracy of the proposed method by comparing its predictions with those obtained by Wood's approach; (2) to further extend the solution to a viscoelastic medium retained by walls that are rigid but elastically constrained against rotation at their base; and (3) to provide numerical data that elucidate the underlying response mechanisms and the effects and relative importance of the numerous parameters involved. The rotationally constrained system is examined as a first step toward a more refined study of the effects of wall flexibility on the response of tanks containing a viscoelastic solid.

In addition to the characteristics of the ground motion and the physical properties of the retained material, the problem parameters considered include the aspect ratio of the system and the rotational flexibility of the wall constraints. Both harmonic and transient ground motions are examined. The response quantities studied include the maximum values of the dynamic wall pressures and of the associated base shear and base moment. 


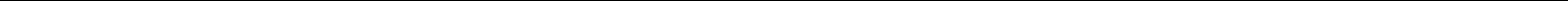




\section{SECTION 2}

\section{SYSTEM AND METHOD OF ANALYSIS}

\subsection{System Considered}

The system investigated is shown in Figure 2.1(a). It is an infinitely long, uniform viscoelastic solid of width $\mathrm{L}$ and height $\mathrm{H}$ which is free at its upper surface, is fully bonded to a rigid base, and is retained along its long vertical boundaries by rigid walls that are elastically constrained against rotation at their base. The stiffness per unit of wall length of each rotational constraint is denoted by $R_{\theta}$. The bases of the layer and the walls are presumed to be excited by a space-invariant, horizontal motion, the acceleration of which at any time $t$ is $\ddot{x}_{g}(t)$ and its maximum value is $\ddot{X}_{g}$. Damping for both the retained material and the rotational constraint is considered to be of the constant hysteretic type.

The properties of the layer are defined by its mass density $\rho$, shear modulus of elasticity $G$, Poisson's ratio $v$, and the material damping factor $\delta$, which is considered to be frequency-independent and the same for both shearing and axial deformations. The latter factor is the same as the $\tan \delta$ factor used by Veletsos and his associates in studies of foundation dynamics and soil-structure interaction (e.g. References $7,8,9$ ), and twice as large as the percentage of critical damping used by other authors in related studies (e.g. References $10,11,12$ ). The damping factor for each wall constraint is denoted by $\delta_{\mathrm{w}}$.

The displacements relative to the moving base and the resulting wall pressures and forces for the system considered can be shown to be identical to those for the system displayed in Figure 2.1(b), which is fixed at its base and is excited by uniform lateral body forces of intensity $-\rho \ddot{x}_{g}(t)$. For exciting frequencies that are small compared to the fundamental frequency of the system, the action of the force-excited system may be easier to visualize than that of the base-excited system.

\subsection{Governing Equations and Assumptions}

The equation of motion for the system is taken in the approximate form proposed by Arias et al [4] as

$$
\psi_{o}^{2} G^{*} \frac{\partial^{2} u}{\partial \xi^{2}}+G^{*} \frac{\partial^{2} u}{\partial \eta^{2}}-\rho H^{2} \frac{\partial^{2} u}{\partial t^{2}}=\rho H^{2} \ddot{x}_{g}
$$

where $\mathrm{u}$ is the horizontal displacement relative to the moving base of a point in the medium defined by 
the cartesian coordinates $\mathrm{x}$ and $\mathrm{y} ; \xi=\mathrm{x} / \mathrm{H}$ and $\eta=\mathrm{y} / \mathrm{H}$ are dimensionless measures of these coordinates; $\mathrm{G}^{*}$ is the complex-valued shear modulus

$$
\mathrm{G}^{*}=\mathrm{G}(1+\mathrm{i} \delta)
$$

where $\mathrm{i}=\sqrt{-1}$; and

$$
\Psi_{0}=\sqrt{\frac{2}{1-v}}
$$

Equation (1) is based on the assumptions that there are no dynamic vertical stresses anywhere in the medium, and that the horizontal variation of the dynamic vertical displacements is negligible so that the shearing stresses $\tau_{x y}$ may be related to the displacements $u$ by

$$
\tau_{\mathrm{xy}}=\mathrm{G}^{*} \frac{1}{\mathrm{H}} \frac{\partial \mathrm{u}}{\partial \eta}
$$

The horizontal normal stresses $\sigma_{\mathrm{x}}$ are related to $\mathrm{u}$ by

$$
\sigma_{x}=\frac{E^{*}}{1-v^{2}} \frac{1}{H} \frac{\partial u}{\partial \xi}=\psi_{0}^{2} G^{*} \frac{1}{H} \frac{\partial u}{\partial \xi}
$$

where $E^{*}=2(1+v) G^{*}$ is the complex-valued Young's modulus of elasticity for the medium. For a more detailed account of the assumptions underlying equation (1) and their rationale, and of the relationship of this equation to others that have been proposed for this problem, the reader is referred to Veletsos and Younan $[5,6]$.

The sign convention for stresses and displacements is that used in theory of elasticity. Specifically, $u$ is positive when directed along the positive direction of the corresponding coordinate axis, $\sigma_{x}$ is positive when it induces tension, and the positive directions of $\tau_{x y}$ are indicated by the inset diagram in Figure 2.1(a).

Equation (1) is solved subject to the boundary conditions

$$
\left.\mathrm{u}\right|_{\eta=0}=0 \quad \text { and }\left.\quad \frac{\partial \mathrm{u}}{\partial \eta}\right|_{\eta=1}=0
$$

of which the second expresses the vanishing of the shearing stresses at the upper surface, and

$$
\left.\mathrm{u}\right|_{\xi=0}=\left.\mathrm{u}\right|_{\xi=L H}=\theta \eta \mathrm{H}
$$

where the wall rotation $\theta$ is considered to be positive when clockwise, and it is assumed that there is no separation between the walls and the retained material. The wall rotation is determined by satisfying the equilibrium of moments about the wall base, i.e. requiring that

$$
M_{b}^{p}-S_{w} \ddot{X}_{g}-I_{w} \ddot{\theta}=R_{\theta}^{*} \theta
$$


where $R_{\theta}^{*}=R_{\theta}\left(1+i \delta_{w}\right)$ is the complex-valued stiffness per unit of wall length of each rotational constraint; $\mathrm{M}_{\mathrm{b}}^{\mathrm{p}}$ represents the base moment per unit of wall length due to the pressures induced by the contained material; and the remaining terms on the left represent the contributions of the wall inertia. The quantities $S_{w}$ and $I_{w}$ represent the first and second mass moments about the wall base, defined by

$$
S_{w}=H^{2} \int_{0}^{1} \mu(\eta) \eta d \eta
$$

and

$$
I_{w}=H^{3} \int_{0}^{1} \mu(\eta) \eta^{2} d \eta
$$

where $\mu(\eta)$ is the mass per unit of area of the wall.

It should be noted that the equilibrium of vertical forces is not satisfied in this approach.

\subsection{Solution for Harmonic Response}

For a harmonic base motion of acceleration

$$
\ddot{x}_{g}(t)=\ddot{X}_{g} \mathrm{e}^{i \omega t}
$$

where $\ddot{X}_{\mathrm{g}}$ is the acceleration amplitude and $\omega$ is the circular frequency of the motion, the steady-state relative displacement $u=u(\xi, \eta, t)$ may be expressed in the form

$$
u(\xi, \eta, t)=\sum_{n=1,3}^{\infty} U_{n}(\xi) \sin \frac{n \pi}{2} \eta e^{i \omega t}
$$

in which $U_{n}(\xi)$ are complex-valued functions of $\xi$, and $n$ is an odd integer. Note that equation (12) satisfies the boundary conditions defined by equation (6).

On substituting equations (11) and (12) into equation (1), and expanding $\ddot{X}_{\mathrm{g}}$ in the form

$$
\ddot{X}_{g}=\frac{4}{\pi} \ddot{X}_{g} \sum_{n=1,3}^{\infty} \frac{1}{n} \sin \frac{n \pi}{2} \eta
$$

it is found that $U_{n}$ must satisfy the ordinary differential equation

$$
\frac{d^{2} U_{n}}{d \xi^{2}}-\alpha_{n}^{2} U_{n}=\frac{4}{n \pi} \frac{\rho \ddot{X}_{g} H^{2}}{\psi_{0}^{2} G^{*}}
$$

in which 


$$
\begin{aligned}
& \alpha_{\mathrm{n}}=\frac{\mathrm{n} \pi}{2 \psi_{0}} \sqrt{\frac{1-\phi_{\mathrm{n}}^{2}+\mathrm{i} \delta}{1+\mathrm{i} \delta}} \\
& \phi_{\mathrm{n}}=\frac{1}{\mathrm{n}} \frac{\omega}{\omega_{1}}
\end{aligned}
$$

and $\omega_{1}$ represents the fundamental circular frequency of the unconstrained medium when it is considered to act as a cantilever shear-beam. The latter frequency is given by

$$
\omega_{1}=\frac{\pi}{2} \frac{v_{s}}{H}
$$

where $v_{s}=\sqrt{G / \rho}$ is the shear wave velocity of the medium.

The solution of equation (14) is given by

$$
\mathrm{U}_{\mathrm{n}}(\xi)=\mathrm{A}_{\mathrm{n}} \cosh \alpha_{\mathrm{n}} \xi+\mathrm{B}_{\mathrm{n}} \sinh \alpha_{\mathrm{n}} \xi-\frac{16}{\pi^{3}} \frac{1}{\mathrm{n}^{3}} \frac{1}{1-\phi_{\mathrm{n}}^{2}+\mathrm{i} \delta} \frac{\rho \ddot{\mathrm{X}}_{\mathrm{g}} \mathrm{H}^{2}}{\mathrm{G}}
$$

where the integration constants $A_{n}$ and $B_{n}$ are determined from equations (7) by expanding $\eta$ in the form

$$
\eta=\frac{8}{\pi^{2}} \sum_{n=1,3}^{\infty} \frac{(-1)^{\frac{n-1}{2}}}{n^{2}} \sin \frac{n \pi}{2} \eta
$$

and expressing $\theta$ as

$$
\theta=\Theta \mathrm{e}^{\mathrm{i} \omega \mathrm{t}}
$$

where $\Theta$ is a complex-valued amplitude. The resulting expression for the displacement $u$ is

$$
u=-\frac{16}{\pi^{3}} \frac{\rho \ddot{X}_{g} H^{2}}{G} \sum_{n=1,3}^{\infty}\left[\frac{1}{n^{3}} \frac{1-f_{n}(\xi)}{1-\phi_{n}^{2}+i \delta}-\frac{\pi}{2} \frac{(-1)^{\frac{n-1}{2}}}{n^{2}} f_{n}(\xi) \frac{G \Theta}{\rho \ddot{X}_{g} H}\right] \sin \frac{n \pi}{2} \eta e^{i \omega t}
$$

and that for the dynamic wall pressure, $\sigma_{\mathrm{w}}=\left.\sigma_{\mathrm{x}}\right|_{\xi=0}$, is

$$
\sigma_{w}=-\frac{8}{\pi^{2}} \psi_{0} \rho \ddot{X}_{g} H \sum_{n=1,3}^{\infty}\left[\frac{1}{n^{2}} a_{n}+\frac{\pi}{2} \frac{(-1)^{\frac{n-1}{2}}}{n} b_{n} \frac{G \Theta}{\rho \ddot{X}_{g} H}\right] \sin \frac{n \pi}{2} \eta e^{i \omega t}
$$

in which 


$$
\begin{aligned}
& f_{n}(\xi)=\frac{\cosh \left[\alpha_{n}\left(\frac{L}{2 H}-\xi\right)\right]}{\cosh \frac{\alpha_{n} L}{2 H}} \\
& a_{n}=\sqrt{\frac{1+i \delta}{1-\phi_{n}^{2}+i \delta}} \tanh \frac{\alpha_{n} L}{2 H}
\end{aligned}
$$

and

$$
b_{n}=\sqrt{(1+i \delta)\left(1-\phi_{n}^{2}+i \delta\right)} \tanh \frac{\alpha_{n} L}{2 H}
$$

The total force or base shear per unit length of each wall, $Q_{b}^{p}$, and the corresponding bending moment, $M_{b}^{p}$, are determined by integration to be

$$
\begin{aligned}
Q_{b}^{p} & =H \int_{0}^{1} \sigma_{w}(\eta) d \eta \\
& =-\frac{16}{\pi^{3}} \psi_{o} \rho \ddot{X}_{g} H^{2} \sum_{n=1,3}^{\infty}\left[\frac{1}{n^{3}} a_{n}+\frac{\pi}{2} \frac{(-1)^{\frac{n-1}{2}}}{n^{2}} b_{n} \frac{G \Theta}{\rho \ddot{X}_{g} H}\right] e^{i \omega t}
\end{aligned}
$$

and

$$
\begin{aligned}
M_{b}^{p} & =H^{2} \int_{0}^{1} \sigma_{w}(\eta) \eta d \eta \\
& =-\frac{32}{\pi^{4}} \Psi_{o} \rho \ddot{X}_{g} H^{2} \sum_{n=1,3}^{\infty}\left[\frac{(-1)^{\frac{n-1}{2}}}{n^{4}} a_{n}+\frac{\pi}{2} \frac{1}{n^{3}} b_{n} \frac{G \Theta}{\rho \ddot{X}_{g} H}\right] e^{i \omega t}
\end{aligned}
$$

These forces are considered to be positive when caused by positive or tensile wall pressures. Specifically, a shearing force at the base of the left wall is positive when directed along the positive $x$ axis, and the corresponding moment is positive when clockwise.

The as yet undetermined rotation amplitude $\Theta$ in equations (26) and (27) is determined from equation (8) by making use of equation (20), with the result

$$
\Theta=\frac{\frac{32}{\pi^{4}} \Psi_{0} \sum_{n=1,3}^{\infty} \frac{(-1)^{\frac{n-1}{2}}}{n^{4}} a_{n}+\frac{S_{w}}{\rho H^{3}}}{\frac{16}{\pi^{3}} \Psi_{0} \sum_{n=1,3}^{\infty} \frac{1}{n^{3}} b_{n}+\frac{\pi^{2}}{4}\left(\frac{\omega}{\omega_{1}}\right)^{2} \frac{I_{w}}{\rho H^{4}}-\frac{R_{\theta}^{*}}{G^{2}}} \frac{\rho \ddot{X}_{g} H}{G}
$$

Equations (26) and (27) define the effects of the dynamic wall pressures, with no provision for the effects of the wall inertia. The total base shear per unit length of each wall, $Q_{b}$, including the contribution of the wall inertia is given by 


$$
Q_{b}=Q_{b}^{p}+\left[-\frac{m_{w}}{\rho H^{2}}+\frac{\pi^{2}}{4}\left(\frac{\omega}{\omega_{1}}\right)^{2} \frac{S_{w}}{\rho H^{3}} \frac{G \Theta}{\rho \ddot{X}_{g} H}\right] \rho \ddot{X}_{g} H^{2} e^{i \omega t}
$$

where $\mathrm{m}_{\mathrm{w}}$ is the total mass of each wall, defined by

$$
\mathrm{m}_{\mathrm{w}}=\mathrm{H} \int_{0}^{1} \mu(\eta) \mathrm{d} \eta
$$

The corresponding base moment, $\mathrm{M}_{\mathrm{b}}$, can most conveniently be determined from

$$
M_{b}=R_{\theta}^{*} \Theta e^{i \omega t}
$$

Equations (22) through (30) reveal that the dynamic pressures and base forces for each wall are functions of the following parameters:

1. The aspect ratio $\mathrm{L} / \mathrm{H}$;

2. The dimensionless measure of the rotational flexibility of the wall constraint

$$
\mathrm{d}_{\theta}=\frac{\mathrm{GH}^{2}}{\mathrm{R}_{\theta}}
$$

3. The mass and inertia ratios $\mathrm{m}_{\mathrm{w}} / \rho \mathrm{H}^{2}, \mathrm{~S}_{\mathrm{w}} / \rho \mathrm{H}^{3}$ and $\mathrm{I}_{\mathrm{w}} / \rho \mathrm{H}^{4}$;

4. Poisson's ratio for the contained material $v$;

5. The damping factors for the contained material and each wall constraint, $\delta$ and $\delta_{w}$, respectively; and

6. The temporal characteristics of the forcing function, which, for a harmonic excitation, are defined by the frequency ratio $\phi_{1}=\omega / \omega_{1}$.

It is important to note that, for the solutions presented here, the horizontal variations of all effects are expressed in closed forms. By contrast, in Wood's more precise analysis, these variations are expressed by trigonometric series, and generally require rather large numbers of terms for their precise definition, particularly for systems with large values of $\mathrm{L} / \mathrm{H}$. This matter is considered further in later sections.

\subsection{Foundation Moment}

For the design of the tank foundation, one must also know the total overturning moment transmitted to it. This moment is clearly equal to the sum of the moments at the base of the walls plus the moment induced by the dynamic base pressures. Considering that the method of analysis employed presumes the absence of any vertical dynamic pressures, the component of the moment contributed by these pressures cannot be computed. However, the total foundation moment per unit of wall length, $M_{f}$, can be determined directly from the lateral inertia forces as 


$$
M_{f}=-H^{3} \int_{0}^{L / H} \int_{0}^{1} \rho\left(\ddot{x}_{g}+\ddot{u}\right) \eta d \eta d \xi-2\left(I_{w} \ddot{\theta}+S_{w} \ddot{x}_{g}\right)
$$

where $\ddot{x}_{\mathrm{g}}+\ddot{\mathrm{u}}=\ddot{\mathrm{x}}$ represents the absolute horizontal acceleration of an arbitrary point of the retained material; the double integral represents the contribution of the inertia forces for this material; and the remaining two terms represent the contribution of the wall inertia. On making use of equations (8) and (31), the last two terms may also be expressed as the difference of the moments defined by equations (31) and (27).

On differentiating equation (21) twice with respect to time, substituting the resulting expression into equation (33), and integrating, the term involving the double integral, I, can be shown to be given by

$$
I=-\frac{1}{2} \rho \ddot{X}_{\mathrm{g}} L H^{2}\left[1+\frac{32}{\pi^{3}}\left(\frac{\omega}{\omega_{1}}\right)^{2} \sum_{n=1,3}^{\infty}\left(\frac{(-1)^{\frac{n-1}{2}}}{n^{5}} \frac{1-\chi_{n}}{1-\phi_{n}^{2}+i \delta}-\frac{\pi}{2} \frac{\chi_{n}}{n^{4}} \frac{G \Theta}{\rho \ddot{X}_{g} H}\right)\right] e^{i \omega t}
$$

where $\chi_{\mathrm{n}}$ is a dimensionless factor defined by

$$
\chi_{n}=\frac{H}{\alpha_{n} L} \tanh \frac{\alpha_{n} L}{2 H}
$$

With the total foundation moment $M_{f}$ established, the component contributed by the dynamic base pressures may, if desired, be determined by substracting from $M_{f}$ the sum of the moments at the base of the walls, $2 \mathrm{M}_{\mathrm{b}}$.

\subsection{Computation of Transient Response}

The response of the system to an arbitrary transient excitation is evaluated from the harmonic response by the Discrete Fourier Transform (DFT) approach in combination with the Fast Fourier Transform (FFT) algorithm. In the application of this procedure, the duration of the forcing function should be increased by the addition of a sufficiently long band of zeros to eliminate the aliasing errors that may be introduced. For the solutions presented here, the duration of the band was taken equal to either the duration of the forcing function or 10 times the fundamental period of the system considered, whichever was larger. 


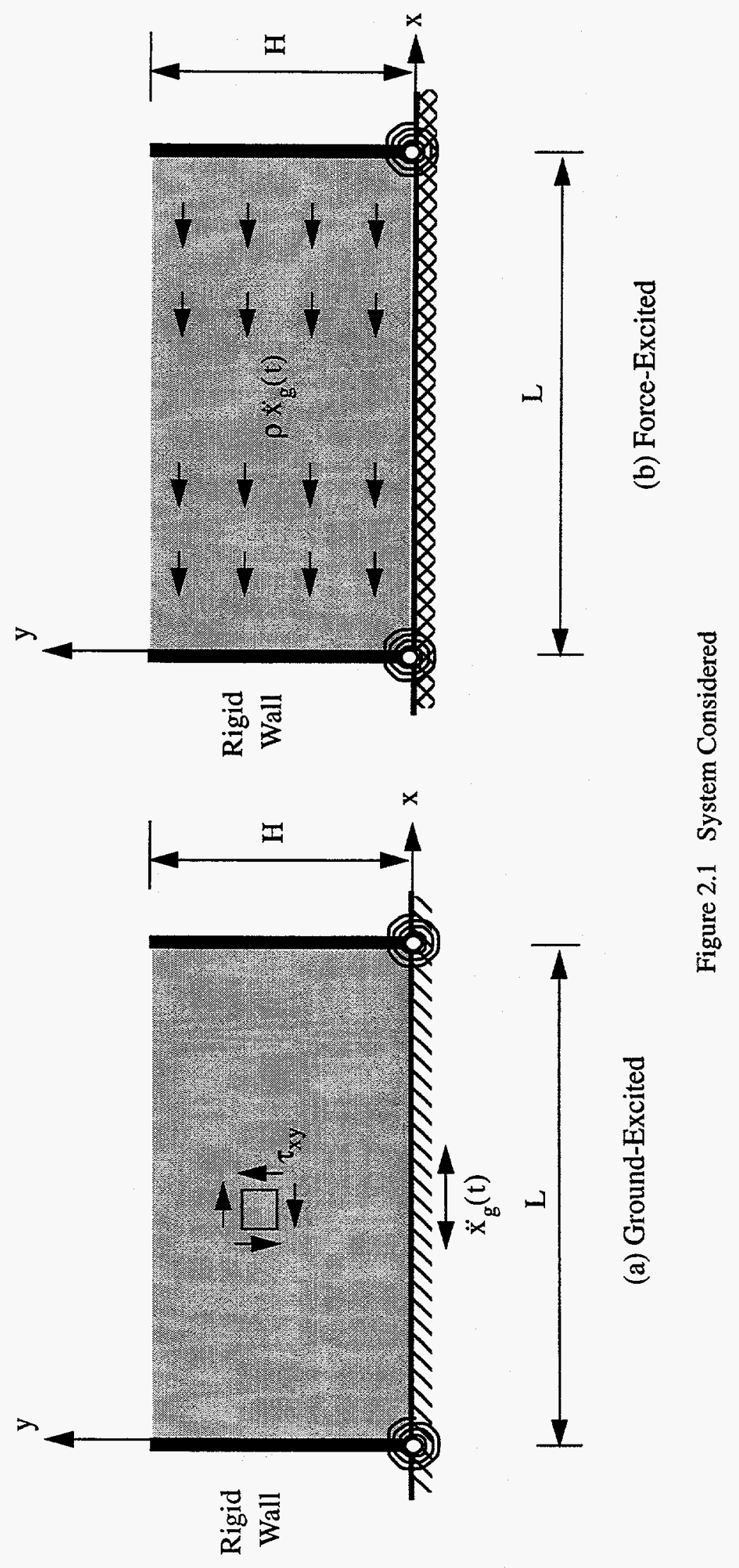




\section{SECTION 3}

\section{RESPONSE OF SYSTEMS WITH FIXED-BASED WALLS}

Consideration is first given to systems for which the walls are fixed against rotation at the base.

\subsection{Static Effects}

It is desirable to begin by examining the effects of excitations the dominant frequencies of which are very small compared to the fundamental frequency of the system (i.e. values of $\phi_{1} \rightarrow 0$ for harmonic ground motions). Such excitations and the resulting effects will be identified with the subscripts st and will be referred to as static, a term which should not be confused with that normally used to represent the effects of gravity forces. In the equivalent, force-excited version of the problem shown in Figure 2.1(b), the static excitation is represented by a set of horizontal body forces of constant intensity $-\rho \ddot{X}_{g}$.

The static values of the base shear and base moment per unit of wall length computed by the proposed simple method of analysis are compared in Figure 3.1 over a range of $\mathrm{L} / \mathrm{H}$ values with those obtained by Wood's exact analysis. The walls in these solutions are presumed to be massless, and Poisson's ratio for the contained material is taken as $v=0.3$. In an effort to define the limiting responses of the system as $\mathrm{L} / \mathrm{H}$ tends to zero and infinity, the results are normalized in two different ways: first, with respect to $\rho \ddot{X}_{g} H L / 2$ for shear and $\rho \ddot{X}_{g} H^{2} L / 4$ for moment; and second, with respect to $\rho \ddot{X}_{g} H^{2}$ for shear and $\rho \ddot{X}_{g} H^{3}$ for moment. The first set of normalizing quantities represents the effects computed on the assumption that all of the contained material acts as a rigid body, whereas the second set of quantities represents those computed on the assumption that only the material within a square adjacent to each wall of side length equal to the wall height acts as a rigid body. As $\mathrm{L} / \mathrm{H} \rightarrow 0$, all of the inertia forces are transmitted horizontally to the walls, and the curves normalized the first way tend to unit values. By contrast, as $\mathrm{L} / \mathrm{H} \rightarrow \infty$, a progressively larger portion of the body forces is transmitted by horizontal shearing action to the base, and the wall forces tend to those obtained for a semi-infinite elastic stratum retained by a single wall. When computed by the simple method of analysis considered here, the latter forces are given by [5]

$$
\left(Q_{b}\right)_{s t}=-0.543 \psi_{o} \rho \ddot{X}_{g} H^{2}=-0.917 \rho \ddot{X}_{g} H^{2}
$$

and 


$$
\left(\mathrm{M}_{\mathrm{b}}\right)_{\mathrm{st}}=-0.325 \psi_{\mathrm{o}} \rho \ddot{\mathrm{X}}_{\mathrm{g}} \mathrm{H}^{3}=-0.549 \rho \ddot{\mathrm{X}}_{\mathrm{g}} \mathrm{H}^{3}
$$

It is clear from Figure 3.1 that the results obtained by the simple approach are in good agreement with Wood's exact results over the range of $\mathrm{L} / \mathrm{H}$ values considered, the agreement being especially good for base moments. These results are also listed in Table 3.1. .

It is convenient and instructive to express the base moment as the product of the base shear and an appropriate height $h$. This height increases from $0.5 \mathrm{H}$ as $\mathrm{L} / \mathrm{H} \rightarrow 0$ to approximately $(2 / \pi) \mathrm{H}$ as $\mathrm{L} / \mathrm{H} \rightarrow \infty$; the latter value represents the centroidal distance for a curve increasing as a quarter-sine from zero at the base to a maximum at the top.

The wall pressures corresponding to the base forces referred to above are plotted for selected values of $\mathrm{L} / \mathrm{H}$ in the left part of Figure 3.2. Although not as good as for the base shear and base moment, the agreement between the approximate and exact solutions is still adequate for most practical purposes. The heightwise variations of the pressures computed by the simplified procedure can more clearly be seen from the plots in the right part of Figure 3.2, where the results are normalized to a unit value at the top. As already intimated, the distributions vary from effectively uniform at low values of $\mathrm{L} / \mathrm{H}$ to one increasing approximately as a quarter-sine as $\mathrm{L} / \mathrm{H} \rightarrow \infty$.

For a massless wall, the static value of the total foundation moment is given by

$$
\left(M_{f}\right)_{s t}=-\frac{1}{2} \rho \ddot{X}_{g} H^{2} L
$$

and the corresponding value of the component contributed by the base pressures, $(\Delta M)_{s t}$, is given by

$$
(\Delta M)_{s t}=\left(M_{f}\right)_{s t}-2\left(M_{b}\right)_{s t}
$$

The horizontal distributions of the base pressures, normalized to unit values at the junctions of the walls and base, are shown in Figure 3.3 for systems with four different values of $\mathrm{L} / \mathrm{H}$. These distributions were determined by Wood's exact method.

\subsection{Results for Harmonic Response}

The real-valued amplitude $\left|Q_{b}\right|$ of the steady-state dynamic base shear per unit of wall length for harmonically excited systems is plotted in Figure 3.4 as a function of the frequency ratio $\omega / \omega_{1}$ for several values of the aspect ratio $\mathrm{L} / \mathrm{H}$ in the range between unity and infinity. As before, the walls in these solutions are presumed to be massless, and Poisson's ratio $v$ and the damping factor $\delta$ of the retained material are taken as 0.3 and 0.1 , respectively. Unless otherwise indicated, the same system parameters are considered for all other solutions that follow. The dashed lines in the figure represent 
the results obtained by the simplified method of analysis, whereas the solid lines represent Wood's exact solutions, all normalized with respect to $\rho \ddot{X}_{\mathrm{g}} \mathrm{H}^{2}$. The latter solutions, which as already noted are defined by double series, are strictly valid only for systems with finite values of $L$. The reported solutions for $\mathrm{L} / \mathrm{H}=\infty$ were determined by taking $\mathrm{L}=50 \mathrm{H}$, a value believed to represent adequately the limiting behavior of the system. These solutions, as well as all others based on Wood's approach, were determined in conformance with the computational guidelines given in Reference 1 , and required consideration of from 13 to 270 terms, the larger number corresponding to systems with the largest value of $\mathrm{L} / \mathrm{H}$ examined. Except for minor differences in the location and magnitude of the highest resonant peak for systems with $\mathrm{L} / \mathrm{H}=1$, the agreement between the exact and approximate solutions in Figure 3.4 is quite satisfactory. The solutions presented in the remainder of this paper are obtained by the simplified approach.

The location and magnitude of the highest resonant peak for each of the frequency response curves displayed in Figure 3.4 are clearly functions of the aspect ratio of the system. For very wide, broad systems with $\mathrm{L} / \mathrm{H} \rightarrow \infty$, this peak occurs at the fundamental frequency of the medium idealized as a cantilever shear beam, and its magnitude, $\left|\mathrm{Q}_{b}\right|_{\max }$, is given $[4,5,6]$ by

$$
\left|Q_{b}\right|_{\max }=\frac{1}{\sqrt{\delta}}\left|\left(Q_{b}\right)_{s t}\right|
$$

which on making use of equation (36) and taking $\delta=0.1$, reduces to $2.9 \rho \ddot{\mathrm{X}}_{\mathrm{g}} \mathrm{H}^{2}$. For the narrower, more slender systems, the peak is reached at the fundamental frequency of the constrained medium, which is naturally higher than that of the unconstrained, and its magnitude first increases and then decreases, reaching a limiting value of

$$
\left|\mathrm{Q}_{\mathrm{b}}\right|_{\max }=\frac{8}{\pi^{2}} \frac{1}{\delta}\left|\left(\mathrm{Q}_{\mathrm{b}}\right)_{\mathrm{st}}\right|
$$

as $L / H \rightarrow 0$, where $\left|\left(Q_{b}\right)_{s t}\right|$ in this case is $\rho \ddot{X}_{g} H L / 2$.

The value of the highest resonant peak may better be appreciated when expressed in the form of an amplification factor, i.e. normalized with respect to the corresponding static value. The variation with $\mathrm{L} / \mathrm{H}$ of the ratio $\left|\mathrm{Q}_{\mathrm{b}}\right|_{\max } /\left|\left(\mathrm{Q}_{\mathrm{b}}\right)_{\text {st }}\right|$ is shown by the solid line in Figure 3.5. Note that this ratio decreases almost continuously from $0.81 / \delta$ for $\mathrm{L} / \mathrm{H} \rightarrow 0$ to $1 / \sqrt{\delta}$ for $\mathrm{L} / \mathrm{H} \rightarrow \infty$. This decreasing trend is due to the combination of the following factors: (1) increasing $\mathrm{L} / \mathrm{H}$ increases the contribution of the higher horizontal modes of vibration of the system relative to that of the fundamental; and (2) for the frequency considered, the dynamic amplification factors for these higher order modes are much lower than that for the fundamental mode. The decreasing trend can also be explained by the fact that, as $\mathrm{L} / \mathrm{H}$ increases, the waves have to travel greater distances before they are reflected by the walls and are therefore affected to a greater extent by damping than would be the case for shorter 
distances or shorter travel times. The rather low resonant peak for the limiting case of $\mathrm{L} / \mathrm{H}=\infty$ is due to the lack of any wave reflections from the far field. The significance of the dashed curves in Figure 3.5 is identified later.

Referring back to Figure 3.4, it should be noted that the higher order peaks of the frequency response curves, which are particularly prominent for values of $\mathrm{L} / \mathrm{H}=10$ and 5 , correspond to the frequencies of the higher order horizontal modes of vibration of the system. For the simplified method of analysis considered, the horizontal displacements of the medium for the mth horizontal and nth vertical participating natural mode are defined by

$$
u(\xi, \eta, t)=C_{m n} \sin m \pi \xi \sin \frac{n \pi}{2} \eta \sin \left(\omega_{m n} t+\varepsilon_{m n}\right)
$$

in which $m$ and $n$ are odd integers; $C_{m n}$ and $\varepsilon_{m n}$ are arbitrary constants; and $\omega_{m n}$ is the associated circular natural frequency of the system, given by

$$
\omega_{\mathrm{mn}}=\omega_{1} \sqrt{\mathrm{n}^{2}+4 \mathrm{~m}^{2} \psi_{0}^{2}(\mathrm{H} / \mathrm{L})^{2}}
$$

As an illustration, it is noted that for $\mathrm{L} / \mathrm{H}=10$ and $v=0.3$, the first four values of $\omega_{\mathrm{m} 1}$, which are $1.056 \omega_{1}, 1.424 \omega_{1}, 1.964 \omega_{1}$ and $2.569 \omega_{1}$, practically coincide with the abscissas of the peaks of the relevant frequency response curve in Figure 3.4. Note further that there is no major peak in this curve corresponding to the frequencies of the higher order vertical natural modes of vibration (i.e. values of $\mathbf{n}>1$ ). It follows that the fundamental vertical mode is the dominant contributor to the response of the system.

The validity of this conclusion can more clearly be seen in Figure 3.6, in which the frequency response curves for base shear computed by the proposed procedure using a sufficiently large number of natural modes are compared with those computed considering the contribution of the fundamental vertical mode only. The excellent agreement between the two solution sets also suggests that the onemode ( $n=1)$ approximation should yield highly accurate results for broad-banded, transient excitations as well. This matter is considered further in a later section.

Comparisons similar to those presented in Figures 3.4 and 3.6 have also been made for the base wall moments and total foundation moment, and they were found to be equally or even more satisfactory.

\subsection{Results for Transient Response}

The solid lines in Figure 3.7 define the amplification factors (ratios of maximum dynamic to static values) of the base shear in the walls of systems subjected to the first $6.3 \mathrm{sec}$ of the N-S component of the ground motion recorded during the El Centro, California earthquake of 1940. The acceleration, 
velocity and displacement traces of this motion are available in Reference 13 and are not reproduced here. The maximum acceleration of the motion is $\ddot{X}_{\mathrm{g}}=0.312 \mathrm{~g}$, and its maximum velocity and displacement are $\dot{X}_{\mathrm{g}}=14.02 \mathrm{in} / \mathrm{sec}$ and $\mathrm{X}_{\mathrm{g}}=8.29 \mathrm{in}$, respectively $(1 \mathrm{in}=2.54 \mathrm{~cm})$. As before, the walls in these solutions are presumed to be massless, and Poisson's ratio and the damping factor for the retained material are taken as $v=0.3$ and $\delta=0.1$. The results are plotted as a function of the fundamental period of the system, $T_{11}=2 \pi / \omega_{11}$, where $\omega_{11}$ is defined by equation (43) with $\mathrm{m}$ and n taken as unity.

The plots in Figure 3.7 are similar to, but by no means the same as, the response spectra for similarly excited, viscously damped single-degree-of-freedom systems. Specifically, for very low-period, stiff media, the amplification factors are effectively unity, and the maximum dynamic values of base shear reduce to the static values listed in Table 3.1. With increasing flexibility of the contained material or increasing period of the system, the amplification factors increase, and after attaining a nearly horizontal plateau, decrease to values which, for highly compliant, soft materials, may be significantly less than unity. An increase in the aspect ratio $\mathrm{L} / \mathrm{H}$ increases the damping capacity of the system and decreases the dynamic amplification factor.

Considering that the fundamental periods of many practical systems fall in the amplified, central region of the plots displayed in Figure 3.7, it is of special interest to examine the largest values of the amplification factors. The variation with $\mathrm{L} / \mathrm{H}$ of the average of the four largest of these factors is shown by the dashed curve in Figure 3.5. As would be expected, these factors are significantly smaller than those for the highest resonant peak of harmonically excited systems, and are less sensitive to variations in the aspect ratio $\mathrm{L} / \mathrm{H}$.

The dashed line in Figure 3.7 defines the amplification factors for systems with $\mathrm{L} / \mathrm{H}=5$ computed by considering the contribution of only the fundamental vertical mode of vibration. As anticipated from the responses of the harmonically excited systems examined earlier, the agreement with the more nearly exact solution is indeed excellent. Considering that the peak values of the dynamic wall pressures for this mode of vibration increase as a quarter-sine from zero at the base to a maximum at the top, and recalling that the distance from the base to the centroid of these pressures is $h=(2 / \pi) H$, the maximum dynamic moment at the base of each wall may be expressed approximately as the product of the corresponding base shear and the indicated height $h$. 
Table 3.1: Static values of base shear, $\left(Q_{b}\right)_{s t}$, and of base moment, $\left(M_{b}\right)_{s t}$, for systems with fixed-based walls; $\mathrm{m}_{\mathrm{w}}=0$ and $v=0.3$.

\begin{tabular}{ccccc}
\hline & \multicolumn{2}{c}{$-\left(\mathrm{Q}_{\mathrm{b}}\right)_{\mathrm{st}} / \rho \ddot{\mathrm{X}}_{\mathrm{g}} \mathrm{H}^{2}$} & \multicolumn{2}{c}{$-\left(\mathrm{M}_{\mathrm{b}}\right)_{\mathrm{st}} / \rho \ddot{\mathrm{X}}_{\mathrm{g}} \mathrm{H}^{3}$} \\
\cline { 2 - 5 } $\mathrm{L} / \mathrm{H}$ & $\begin{array}{c}\text { Present } \\
\text { Approach }\end{array}$ & $\begin{array}{c}\text { Wood's Exact } \\
\text { Approach }\end{array}$ & $\begin{array}{c}\text { Present } \\
\text { Approach }\end{array}$ & $\begin{array}{c}\text { Wood's Exact } \\
\text { Approach }\end{array}$ \\
\hline 0 & $0.5 \rho \ddot{\mathrm{X}}_{\mathrm{g}} \mathrm{HL}$ & $0.5 \rho \ddot{\mathrm{X}}_{\mathrm{g}} \mathrm{HL}$ & $0.25 \rho \ddot{\mathrm{X}}_{\mathrm{g}} \mathrm{H}^{2} \mathrm{~L}$ & $0.25 \rho \ddot{\mathrm{X}}_{\mathrm{g}} \mathrm{H}^{2} \mathrm{~L}$ \\
0.5 & 0.230 & 0.232 & 0.123 & 0.121 \\
1.0 & 0.420 & 0.428 & 0.236 & 0.230 \\
2.0 & 0.682 & 0.699 & 0.399 & 0.393 \\
3.0 & 0.816 & 0.843 & 0.485 & 0.481 \\
4.0 & 0.876 & 0.909 & 0.523 & 0.521 \\
5.0 & 0.901 & 0.936 & 0.539 & 0.538 \\
6.0 & 0.911 & 0.948 & 0.545 & 0.545 \\
8.0 & 0.916 & 0.954 & 0.549 & 0.549 \\
$\infty$ & 0.917 & 0.955 & 0.549 & 0.549 \\
\hline
\end{tabular}




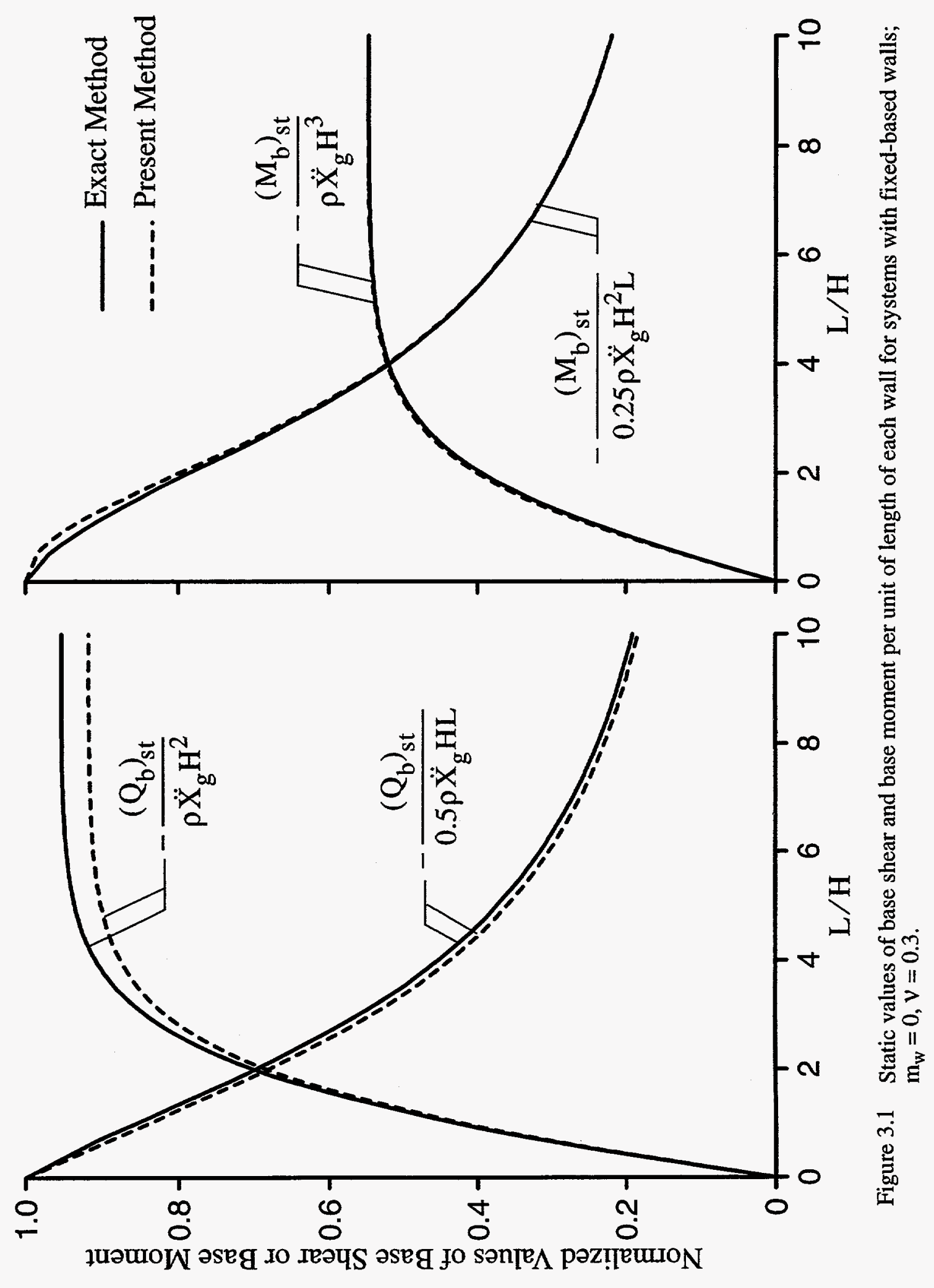




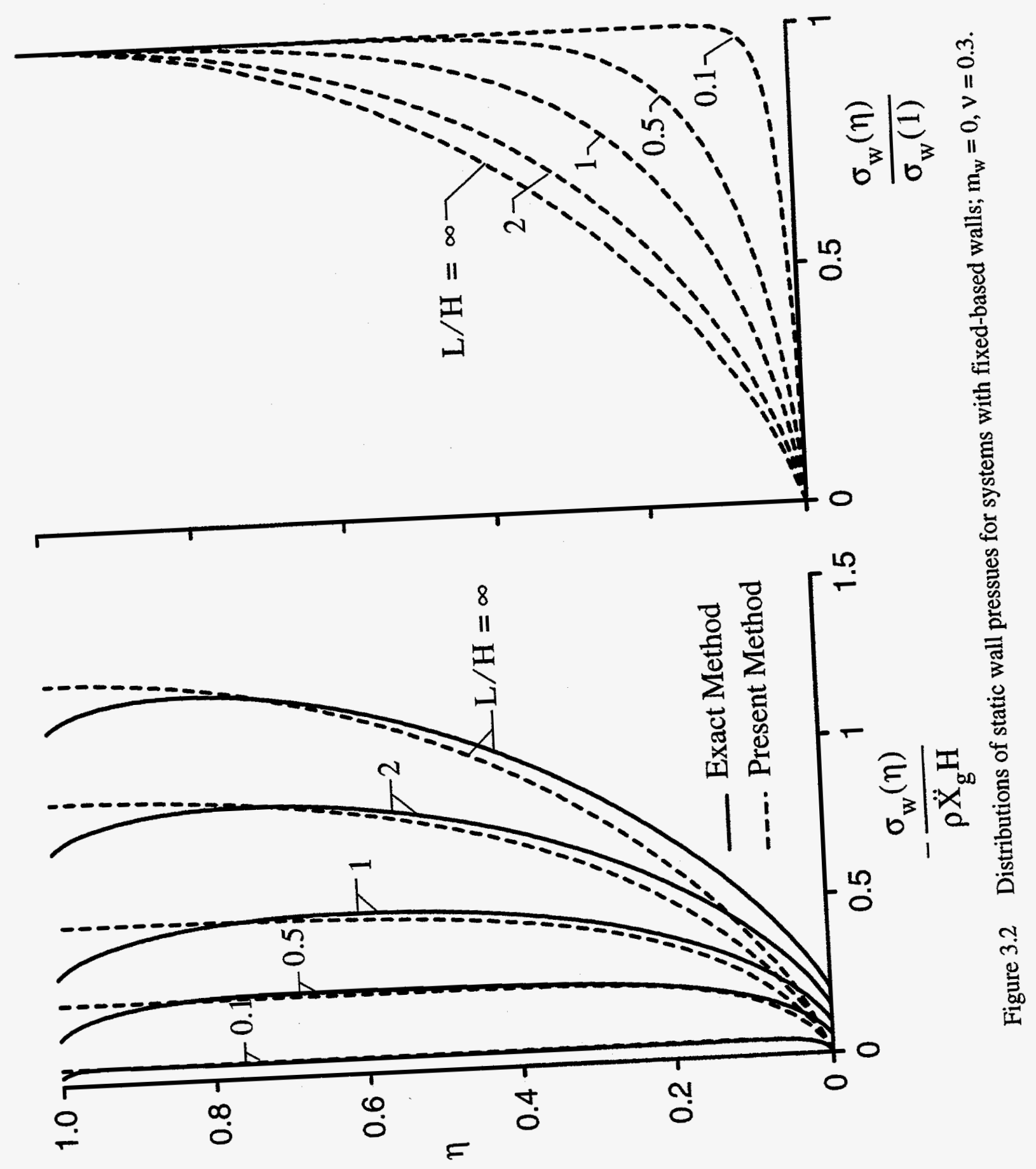




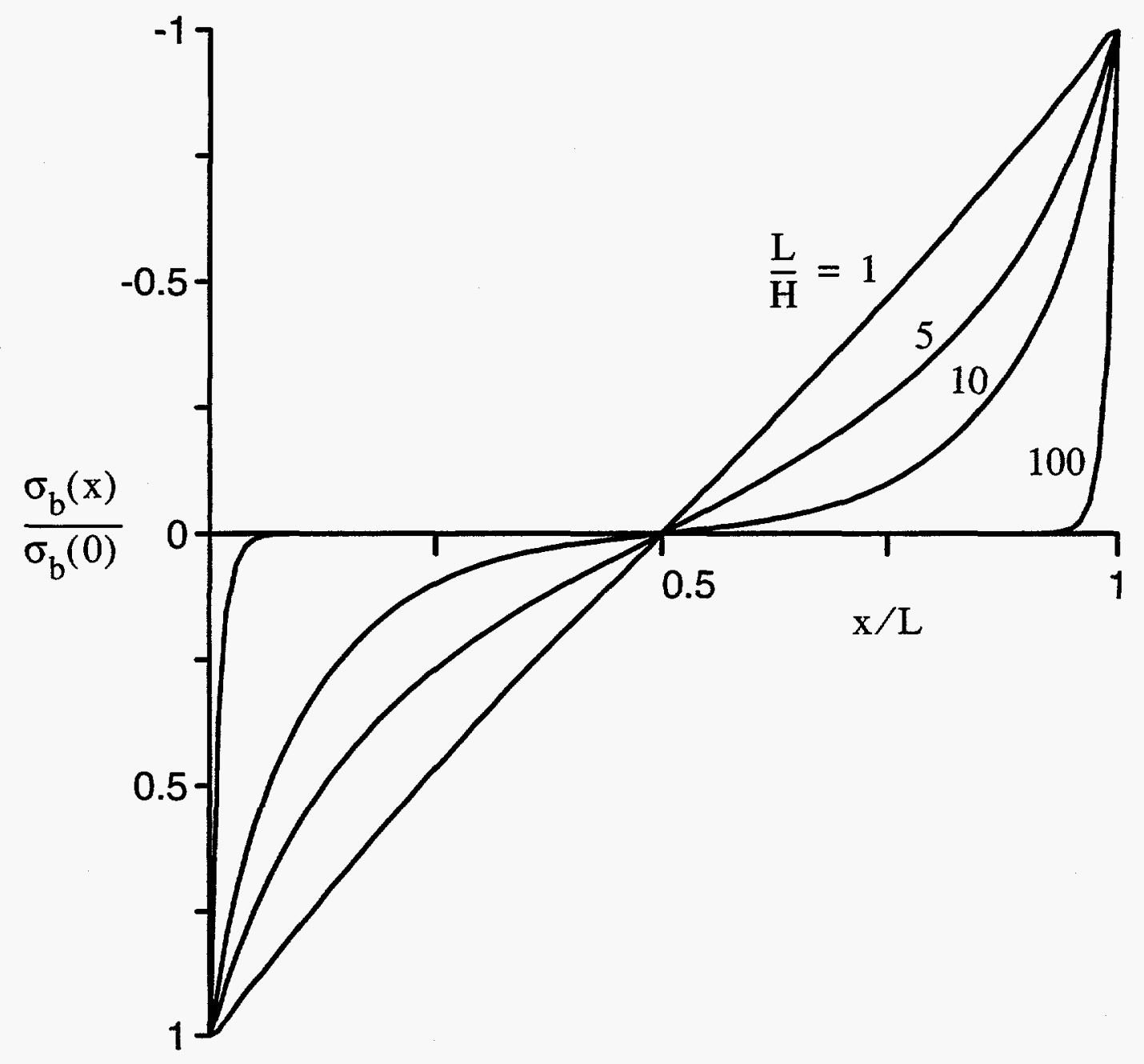

Figure 3.3 Distributions of static base pressures for systems with fixed-based walls; $\mathrm{m}_{\mathrm{w}}=0, \mathrm{v}=0.3$. 

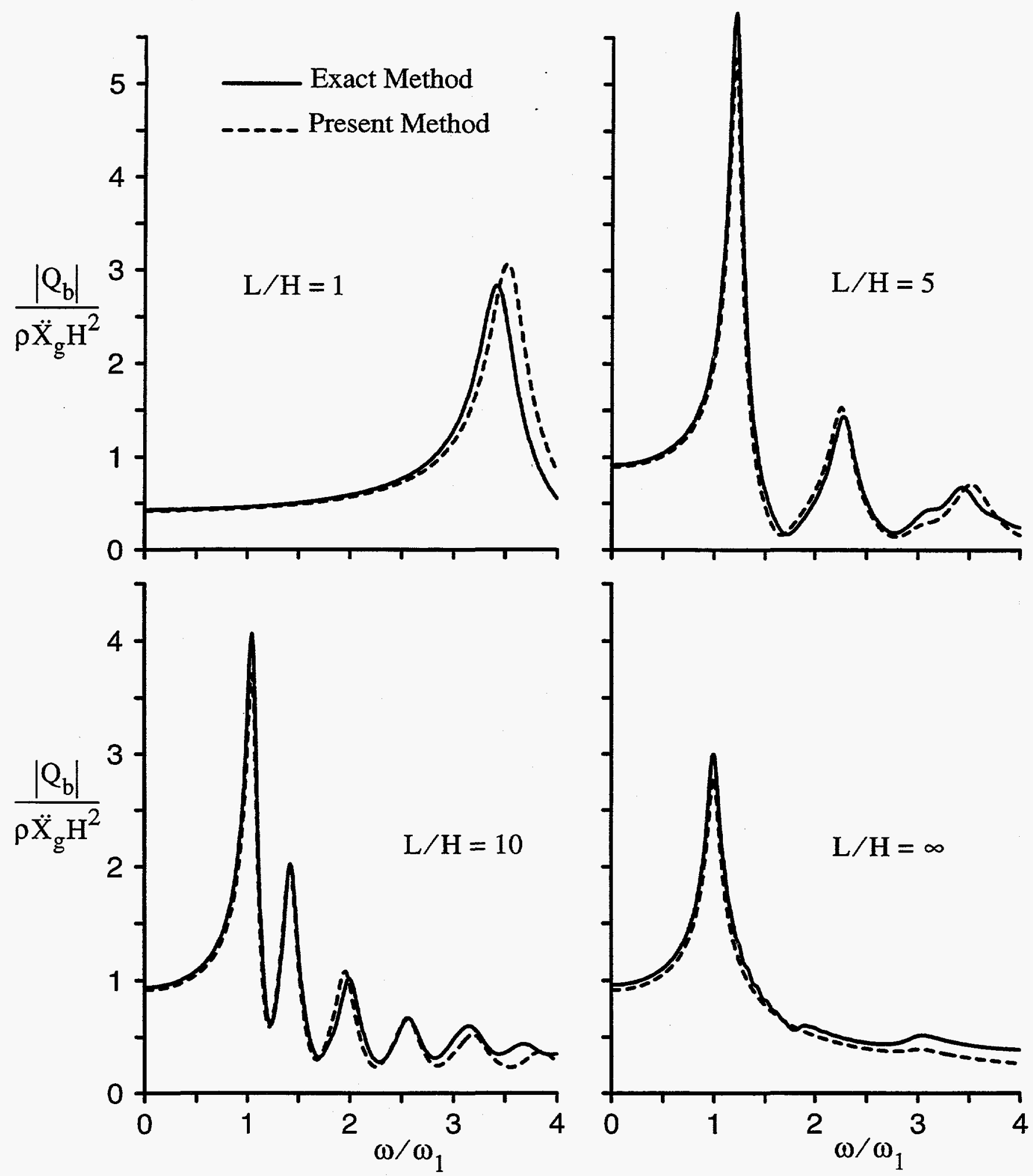

Figure 3.4 Amplitude of base shear per unit of length of each wall for harmonically excited systems with fixed-based walls; $m_{w}=0, v=0.3, \delta=0.1$. 


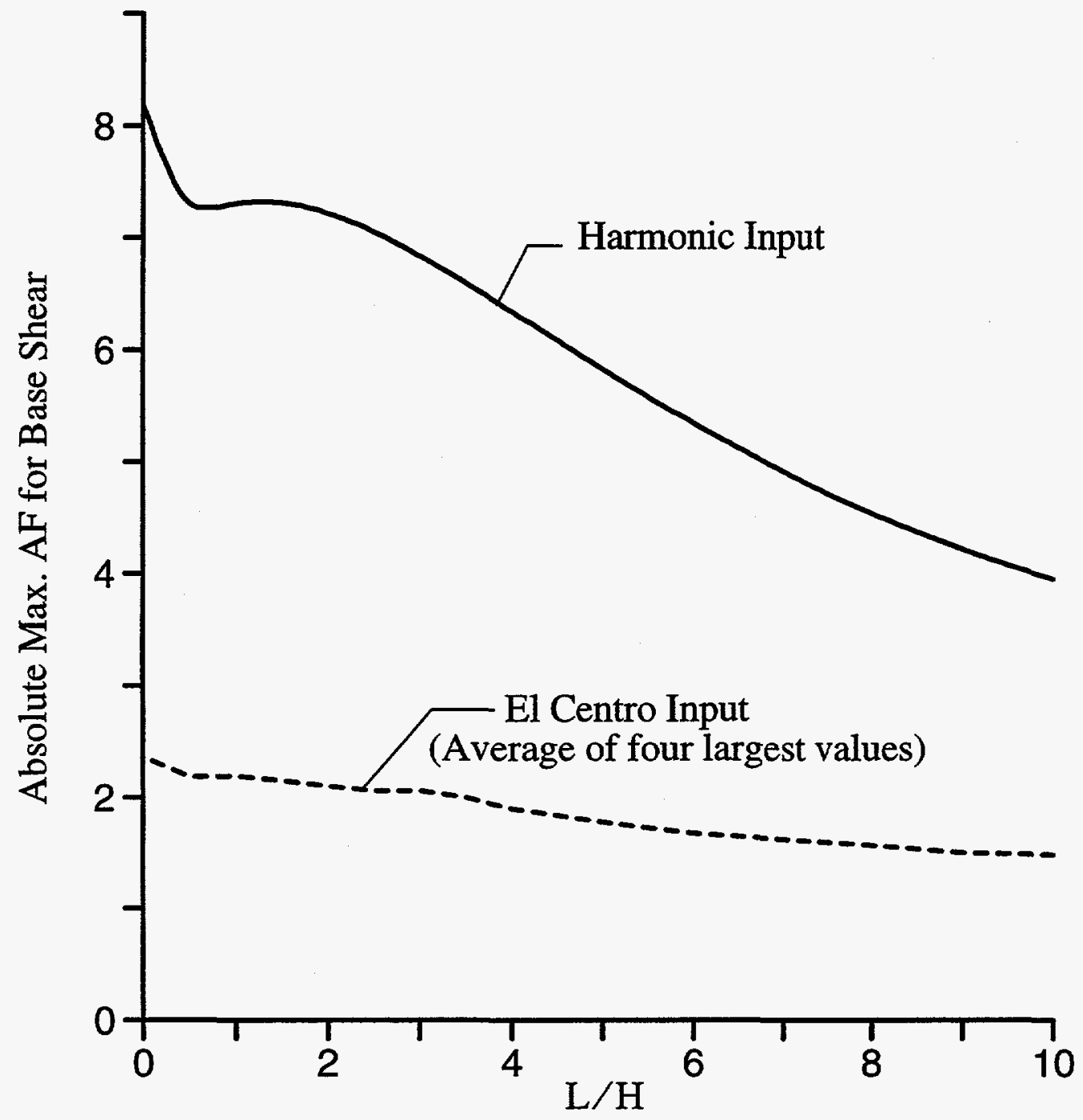

Figure 3.5 Absolute maximum amplification factor for base shear in wall of systems with fixed-based walls subjected to harmonic and transient excitations; $\mathrm{m}_{\mathrm{w}}=0$, $v=0.3, \delta=0.1$. 

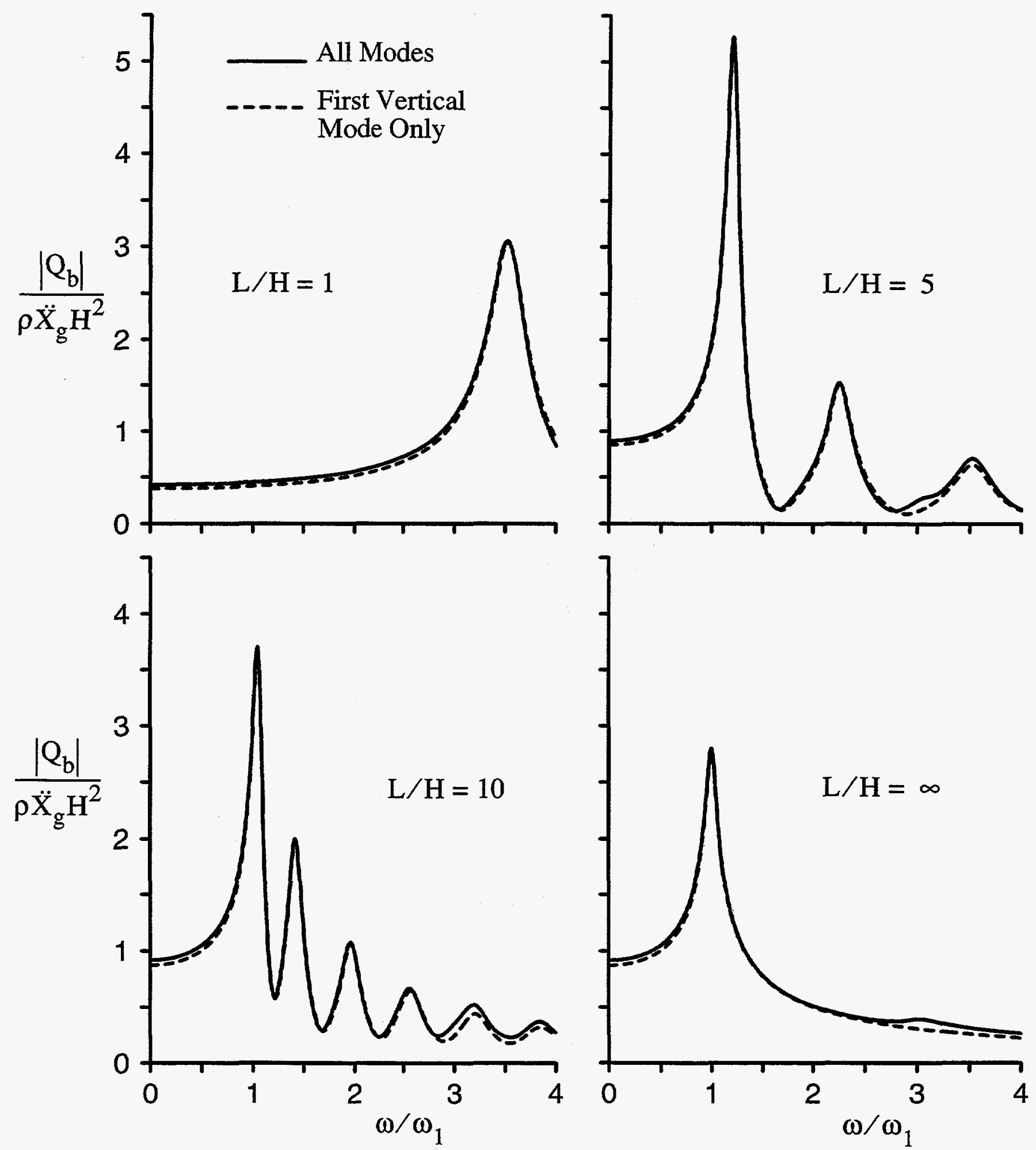

Figure 3.6 Comparison of frequency response curves for base shear in wall of systems computed considering many and a single vertical mode of vibration; $m_{w}=0, v=0.3, \delta=0.1$. 


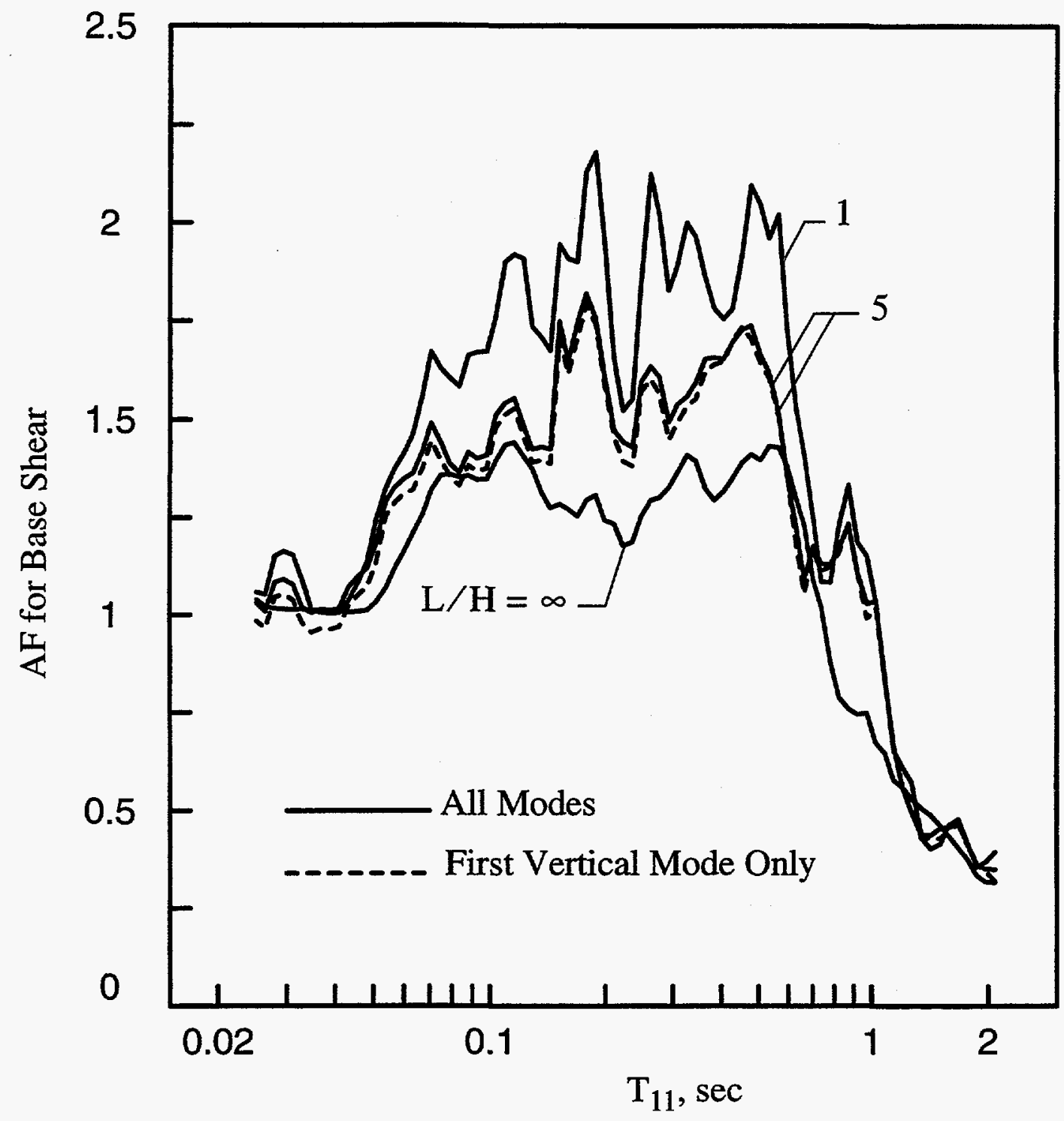

Figure 3-7 Amplification factors for base wall-shear computed for El Centro record considering many and a single mode of vibration; $\mathrm{m}_{\mathrm{w}}=0, v=0.3, \delta=0.1$. 


\section{SECTION 4}

\section{RESPONSE OF SYSTEMS WITH ROTATIONALLY CONSTRAINED WALLS}

The rotational flexibility of the wall base has a two-fold effect: (1) It decreases the horizontal extensional stiffness of the retained material relative to its shearing stiffness, and this reduction decreases the proportion of the inertia forces transmitted by extensional action to the walls and increases the proportion transmitted by shearing action to the base; (2) it modifies the dynamic properties of the system, specifically, its natural frequency and damping capacity. The latter modifications may increase or decrease the dynamic amplification factors of response. It is the purpose of this section to identify the net effect of these changes.

\subsection{Static Effects}

In Figure 4.1(a), the static value of the base shear is plotted as a function of the dimensionless measure of the flexibility of the rotational constraint, $d_{\theta}$. As anticipated, the wall flexibility reduces the magnitude of the base shear, the reduction being generally quite substantial, particularly for the larger values of the aspect ratio.

Figure 4.1(b) shows the corresponding variations of the height $h$ by which the maximum base shear must be multiplied to obtain the maximum base moment. As previously demonstrated for the limiting case of a wall retaining a semi-infinite medium [6], these variations also are substantial and reflect the changed heightwise distribution of the wall pressures. The data displayed in Figure 4.1 are also listed in parts (a) and (b) of Table 4.1.

\subsection{Fundamental Period of System}

The variation with $d_{\theta}$ of the fundamental period of the system, $T_{11}$, is shown in Figure 4.2 for several values of the aspect ratio $\mathrm{L} / \mathrm{H}$. The results are normalized with respect to the $T_{1}=4 \mathrm{H} / \mathrm{v}_{\mathrm{s}}$, the fundamental period of the system computed on the assumption that the retained material behaves as an unconstrained cantilever shear beam. Note that, whereas the period of the semi-infinite medium $(L / H=\infty)$ is independent of $d_{\theta}$ and equal to $T_{1}$, that of the finite-sized system is a function of $d_{\theta}$ and lower than $T_{1}$, the reduction being quite substantial for the narrower, more slender systems. Some of the data displayed in Figure 4.2 are also listed in part (c) of Table 4.1. 


\subsection{Results for Dynamic Response}

The effect of wall flexibility on the response of systems subjected to the El Centro record is shown in Figure 4.3 , in which the absolute maximum value of the base shear for each wall, normalized with respect to $\rho \ddot{X}_{\mathrm{g}} \mathrm{H}^{2}$, is plotted as a function of the fundamental period of the system, $\mathrm{T}_{11}$. Several values of the aspect ratio $L / H$ and of the flexibility factor for the base spring $d_{\theta}$ are considered. As before, the walls are presumed to be massless, and Poisson's ratio and the damping factor for the retained material are taken as $v=0.3$ and $\delta=0.1$. Additionally, the damping factor for the wall is taken as $\delta_{\mathrm{w}}=0.04$ (or 2 percent of critical damping). It is important to note that the period $\mathrm{T}_{11}$, which may be determined readily from the data presented in Figure 4.2 and Table 4.1, is different for systems with different combinations of $\mathrm{L} / \mathrm{H}$ and $\mathrm{d}_{\theta}$.

As would be expected, the results in Figure 4.3 for the extremely low-period, stiff systems are the same as those obtained for the statically excited systems examined in Figure 4.1. Like the static effects, the peak dynamic effects generally decrease with increasing flexibility of the walls, but the reduction is a function of both the aspect ratio $\mathrm{L} / \mathrm{H}$ and the natural period of the system $\mathrm{T}_{11}$. This reduction in dynamic response contradicts the well-established effect of wall flexibility on the response of liquid containing tanks [14]. For the range of parameters normally encountered in practice, the wall flexibility in the latter systems tends to increase rather than decrease their response.

This fundamental difference in the responses of liquid and solid containing tanks may better be appreciated from Figure 4.4, in which the average of the four largest values of the base shear displayed in Figure 4.3 are plotted as a function of $L / H$ for fixed values of $d_{\theta}$. Note that the curves for the elastically constrained systems cross that for the fixed-based systems, and that only for the combination of parameters corresponding to the right of the points identified by heavy dots does the flexibility of the walls reduce the maximum response of the system. For the rather slender systems represented by very small values of $\mathrm{L} / \mathrm{H}$, the horizontal shearing stiffness of the retained material is negligible compared to its extensional stiffness; the material in this case effectively acts as a liquid; and, as would be expected of liquid containing systems, the flexibility of the walls tends to increase the response.

The same trends can more clearly be seen in Figure 4.5 , which presents corresponding plots for the largest resonant peak of harmonically excited systems. The values of $v, \delta$ and $\delta_{w}$ for these solutions are the same as for the solutions presented in Figures 4.3 and 4.4. The peak responses for this motion are naturally substantially higher than for the El Centro, but the interrelationship of the solutions for the elastically constrained and fixed-based walls is quite similar in the two cases.

Also of practical value is the interrelationship of the responses obtained for systems with finite and infinite values of $\mathrm{L} / \mathrm{H}$, the difference reflecting the effects of wall-to-wall interaction. For the static 
condition of loading examined in Figure 3.1, the presence of the second wall decreases the base values of wall shear and wall moment below those obtained for a single wall retaining a semi-infinite stratum (see curves normalized with respect to $\rho \ddot{X}_{\mathrm{g}} \mathrm{H}^{2}$ for shear and with respect to $\rho \ddot{X}_{\mathrm{g}} \mathrm{H}^{3}$ for moment). By contrast, for the dynamically excited systems considered in Figures 4.4 and 4.5, wall-towall interaction increases the response for all but the extremely slender systems, the changes being functions of the flexibility of the wall constraints and of the temporal characteristics of the ground motion.

It is finally instructive to compare the absolute maximum values of the dynamic shears in Figure 4.3 to the corresponding low-period, static values. In part (a) of Figure 4.6, the amplification factors for the average of the 4 largest values displayed in Figure 4.3 are plotted as a function of $\mathrm{L} / \mathrm{H}$ for five values of $d_{\theta}$. Note that these factors increase with decreasing $L / H$, and that they are generally higher for the elastically constrained walls than the fixed-based walls. For the limiting case of $\mathrm{L} / \mathrm{H} \rightarrow 0$, the amplification factors for the system with elastically constrained walls correspond to the damping value of the walls (i.e. $\delta_{w}=0.04$ or 2 percent of critical damping), whereas those for the fixed-based walls correspond to the damping value of the contained material (i.e. $\delta=0.10$ ). By contrast, for $\mathrm{L} / \mathrm{H} \rightarrow \infty$, the amplification factors correspond to those of the semi-infinite medium, and range from $\sqrt{\delta}=0.316$ (or 15.8 percent of critical damping) for the fixed-based walls to $\delta=0.10$ (or 5 percent of critical damping) for the very lightly constrained walls. These trends can more clearly be seen in part (b) of Figure 4.6 which shows the corresponding peak amplification factors of harmonically excited systems.

A Word of Caution. The amplification factors shown in Figure 4.6 apply only to the amplified central region of the plots presented in Figure 4.3; outside this region, they may be significantly lower. In fact, unless the flexibility factor for the wall constraint $d_{\theta}$ is quite high, the fundamental period of the wall-solid system tends to zero as $\mathrm{L} / \mathrm{H} \rightarrow 0$ (see Figure 4.2), the amplification factor then tends to unity, and the maximum dynamic response of the system tends to its static value.

\subsection{Contribution of Higher Modes}

Note has already been taken of the fact that, for fixed-based walls, the maximum values of the base shear and base moment are controlled by the fundamental vertical mode of vibration of the system. On the other hand, for an elastically constrained wall retaining a semi-infinite stratum, it has been shown (see Figure 8 in Reference 6) that the higher order vertical modes of vibration, particularly the second, may be significant contributors to the response, the relative importance of these modes increasing with increasing flexibility of the wall constraint. As might be expected, the same general trends also hold true for the finite-sized systems examined here.

Figure 4.7 compares the normalized values of the maximum base shears induced by the El Centro record in the walls of systems with $L / H=2$. Three values of the wall flexibility parameter $d_{\theta}$ are 
considered, and all other parameters are the same as before. The solid lines represent the results obtained considering many vertical modes of vibration, whereas the dashed lines represent those obtained with only the fundamental vertical mode. Although not shown, the results obtained with the first two vertical modes are in very good agreement with the multimode results over the entire range of parameters examined. It may be recalled that the contributions of all horizontal modes of vibration are accounted for directly in the method of analysis employed.

Considering that the participation of the higher vertical modes of vibration (mainly second) increases with increasing $d_{\theta}$, the height $h$ at which the base shear must be concentrated to yield the correct base moment must decrease with increasing $d_{\theta}$. This trend, which is consistent with that displayed in part (b) of Figure 4.1 for statically excited systems, is shown in Figure 4.8 to hold true for the seismically excited systems as well. It is further observed that the effective height is insensitive to variations in the natural period of the system and that, to a reasonable approximation, it may be taken equal to its static value. 
Table 4.1: Static values of base shear $\left(\mathrm{Q}_{b}\right)_{s t}$, of effective height $h$, and of fundamental period $\mathrm{T}_{11}$ for systems with elastically constrained walls; $\quad v=0.3$ and $\mathrm{m}_{\mathrm{w}}=0$.

\begin{tabular}{lllllll}
\hline $\mathrm{L} / \mathrm{H}$ & $\mathrm{d}_{\theta}=0$ & $\mathrm{~d}_{\theta}=1$ & $\mathrm{~d}_{\theta}=2$ & $\mathrm{~d}_{\theta}=3$ & $\mathrm{~d}_{\theta}=4$ & $\mathrm{~d}_{\theta}=5$ \\
\hline
\end{tabular}

(a) Values of $-\left(Q_{b}\right)_{s t} / \rho \ddot{X}_{g} H^{2}$

$\begin{array}{lll}0.5 & 0.230 & 0.205 \\ 1.0 & 0.420 & 0.337 \\ 2.0 & 0.682 & 0.471 \\ 3.0 & 0.816 & 0.523 \\ 4.0 & 0.876 & 0.544 \\ 5.0 & 0.901 & 0.552 \\ 6.0 & 0.911 & 0.555 \\ 8.0 & 0.916 & 0.557 \\ \infty & 0.917 & 0.557\end{array}$

$0.5 \rho \ddot{X}_{\mathrm{g}} \mathrm{HL} \quad 0.5 \rho \ddot{\mathrm{X}}_{\mathrm{g}} \mathrm{HL}$

$0.5 \rho \ddot{\mathrm{X}}_{\mathrm{g}} \mathrm{HL}$

$0.5 \rho \ddot{X}_{\mathrm{g}} \mathrm{HL}$

$0 \quad 0.5 \rho \ddot{X}_{\mathrm{g}} \mathrm{H}$

$0.5 \rho \ddot{X}_{\mathrm{g}} \mathrm{HL}$

0.187

0.175

0.165

0.158

0.292

0.264

0.244

0.230

0.381

0.332

0.411

0.353

0.301

0.279

0.423

0.360

0.316

0.292

0.427

0.363

0.322

0.296

0.429

0.364

0.324

0.298

0.430

0.365

0.325

0.299

0.430

0.365

0.326

0.300

0.326

0.300

(b) Values of $h / H$

\begin{tabular}{ccccccc}
0 & 0.5 & 0.5 & 0.5 & 0.5 & 0.5 & 0.5 \\
0.5 & 0.536 & 0.490 & 0.451 & 0.417 & 0.389 & 0.363 \\
1.0 & 0.562 & 0.493 & 0.439 & 0.395 & 0.360 & 0.330 \\
2.0 & 0.586 & 0.505 & 0.443 & 0.395 & 0.356 & 0.324 \\
3.0 & 0.594 & 0.510 & 0.447 & 0.398 & 0.358 & 0.326 \\
4.0 & 0.597 & 0.513 & 0.449 & 0.399 & 0.360 & 0.327 \\
5.0 & 0.598 & 0.513 & 0.450 & 0.400 & 0.360 & 0.327 \\
6.0 & 0.598 & 0.514 & 0.450 & 0.400 & 0.360 & 0.328 \\
8.0 & 0.599 & 0.514 & 0.450 & 0.400 & 0.360 & 0.327 \\
$\infty$ & 0.600 & 0.514 & 0.450 & 0.400 & 0.360 & 0.327 \\
& & & (c) Values of $\mathrm{T}_{11} / \mathrm{T}_{1}$ & & \\
& & & & & \\
0.5 & 0.146 & 0.422 & 0.537 & 0.607 & 0.655 & 0.691 \\
1.0 & 0.284 & 0.564 & 0.673 & 0.734 & 0.773 & 0.800 \\
2.0 & 0.509 & 0.721 & 0.801 & 0.843 & 0.869 & 0.886 \\
3.0 & 0.664 & 0.807 & 0.863 & 0.892 & 0.910 & 0.922 \\
4.0 & 0.764 & 0.859 & 0.899 & 0.920 & 0.933 & 0.942 \\
5.0 & 0.829 & 0.893 & 0.922 & 0.938 & 0.948 & 0.954 \\
6.0 & 0.871 & 0.916 & 0.938 & 0.950 & 0.958 & 0.962 \\
8.0 & 0.921 & 0.945 & 0.958 & 0.965 & 0.970 & 0.974 \\
10.0 & 0.947 & 0.962 & 0.969 & 0.974 & 0.978 & 0.980 \\
$\infty$ & 1 & 1 & 1 & 1 & 1 & 1 \\
\hline
\end{tabular}




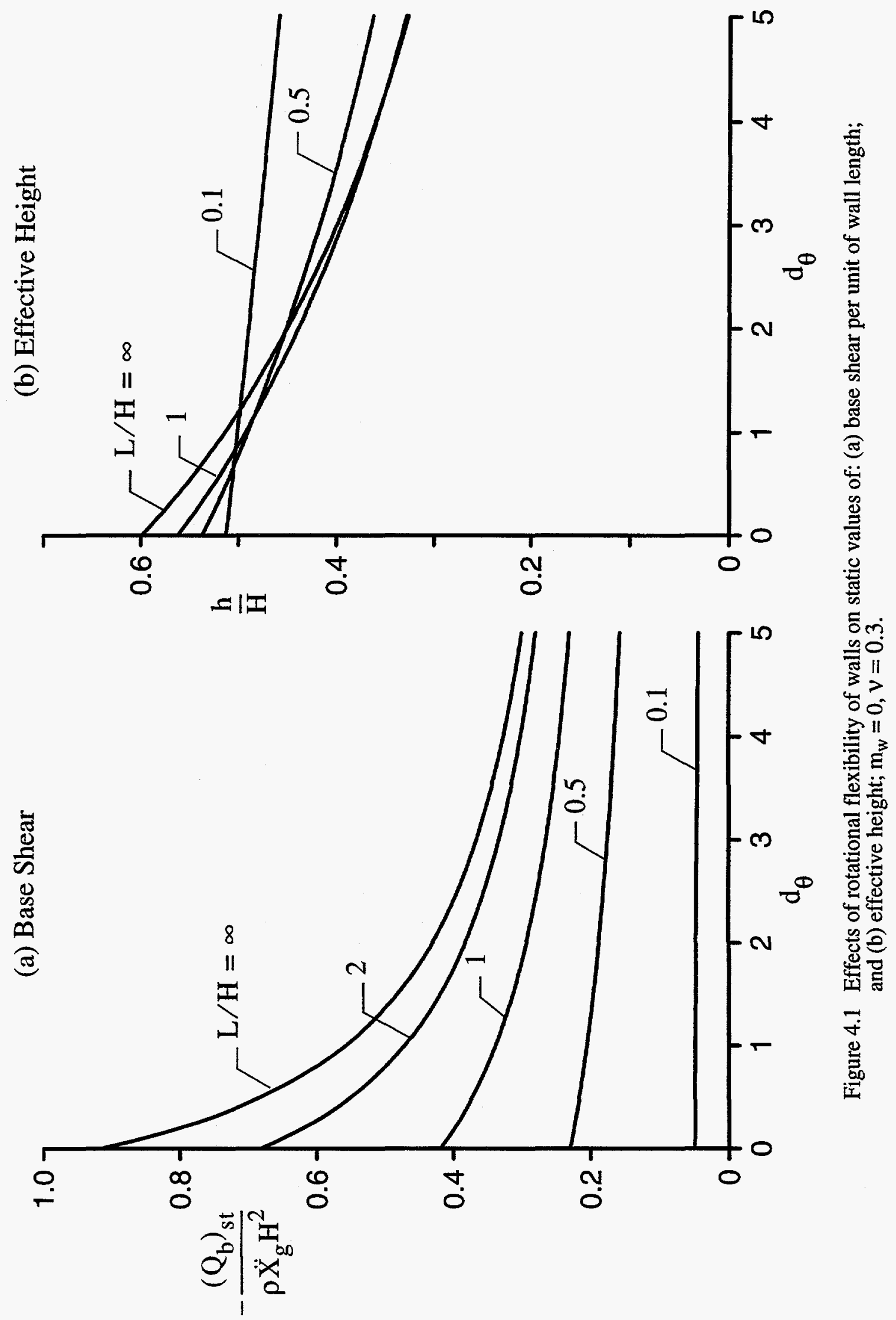




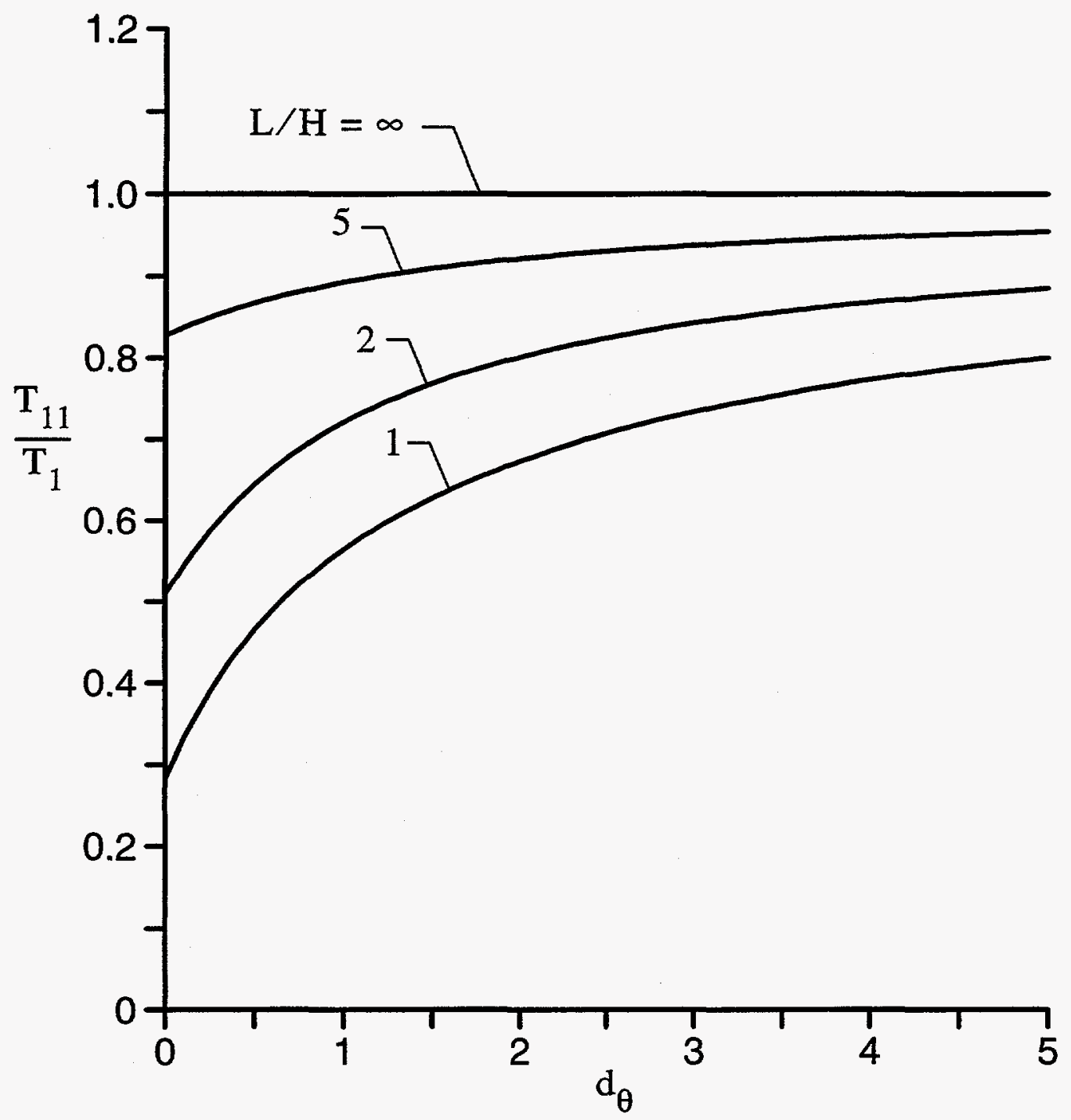

Figure 4.2 Effect of rotational flexibility of wall on fundamental period of vibration of systems with different aspect ratios; $\mathrm{m}_{\mathrm{w}}=0, v=0.3$. 


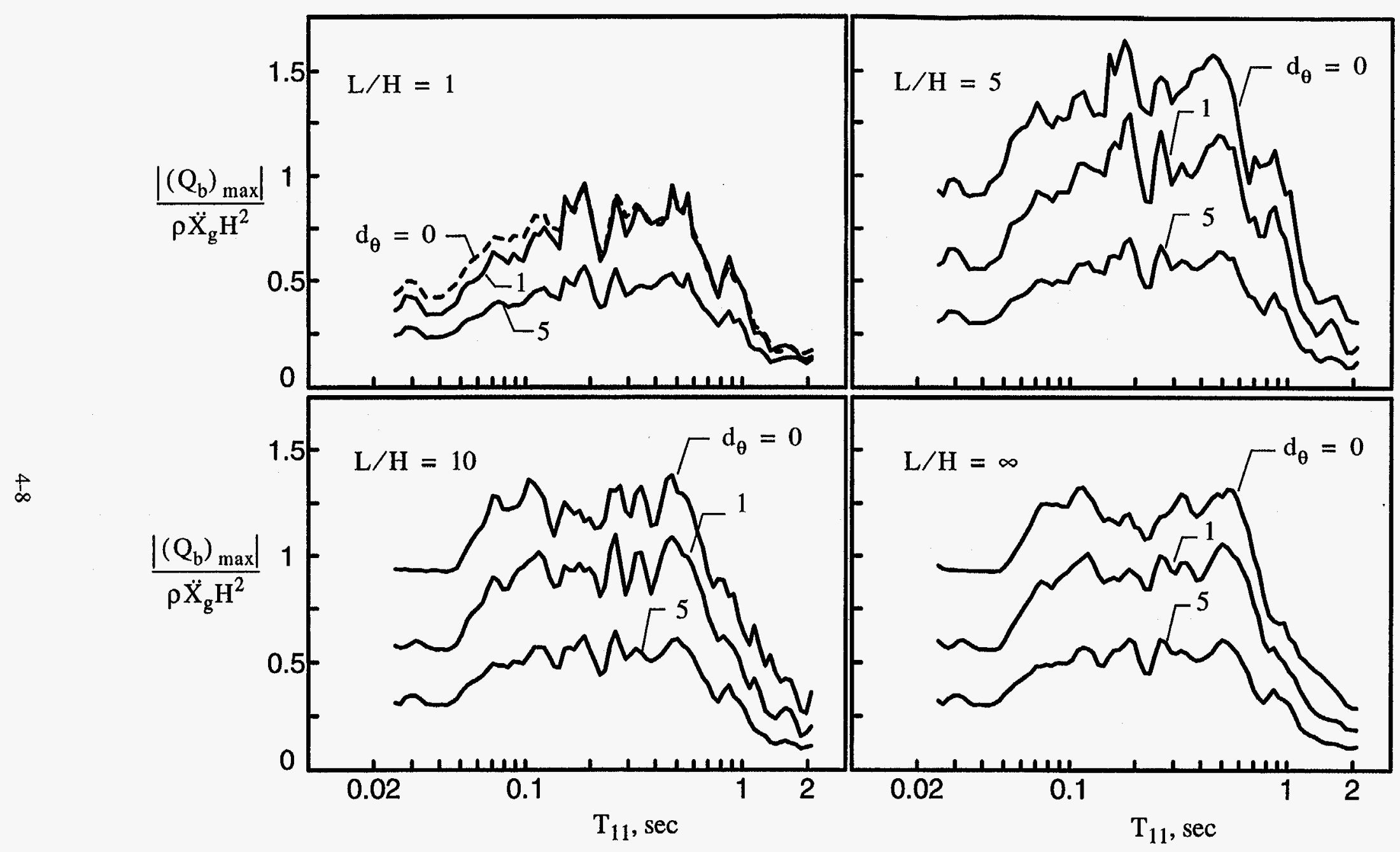

Figure 4.3 Maximum base shear per unit of length of each wall for systems with elastically constrained walls subjected to El Centro record; $\mathrm{m}_{\mathrm{w}}=0, v=0.3, \delta=0.1, \delta_{\mathrm{w}}=0.04$. 


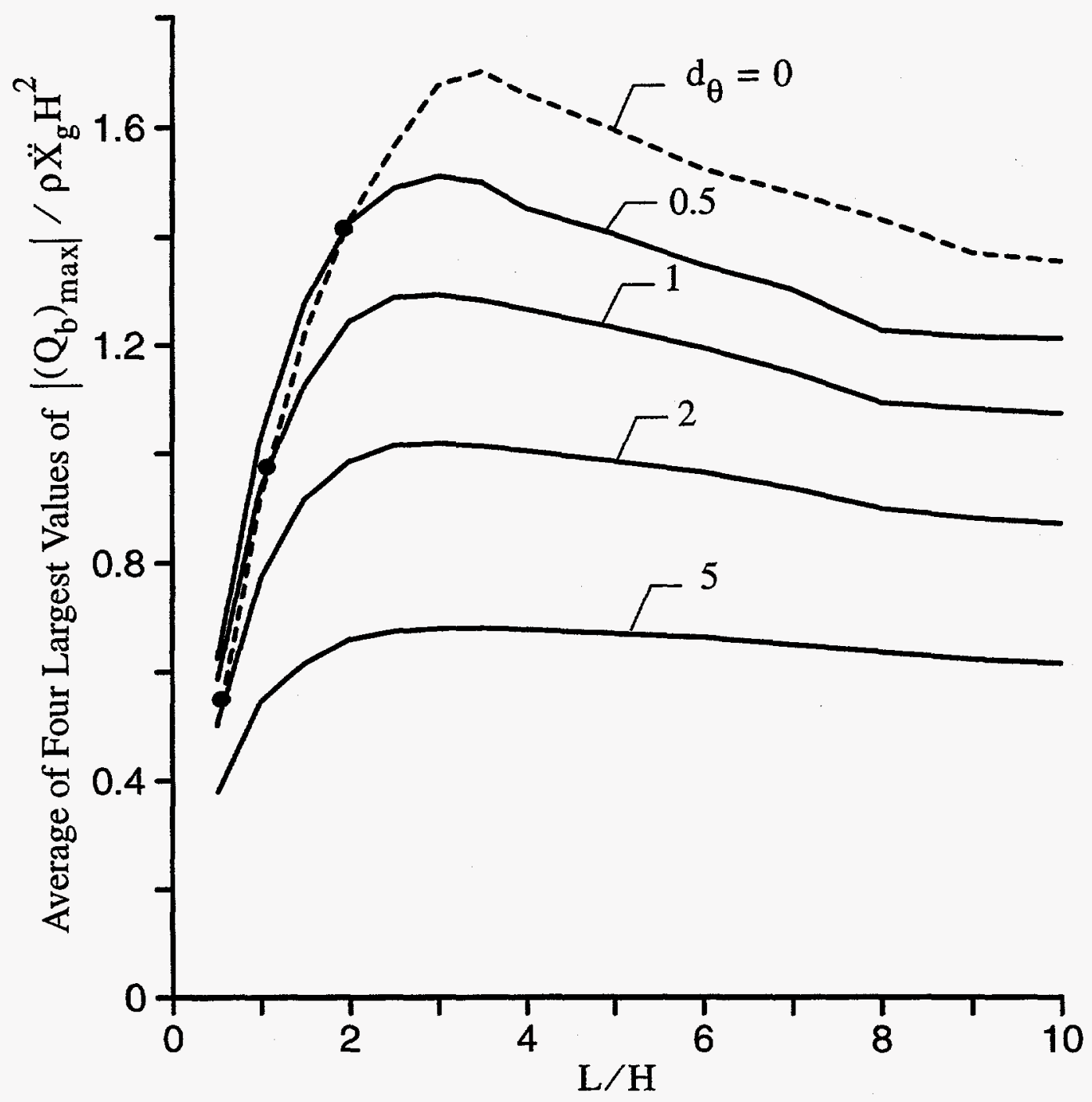

Figure 4.4 Absolute maximum value of base shear per unit of length of each wall for systems with elastically constrained walls subjected to $\mathrm{El}$ Centro record; $\mathrm{m}_{\mathrm{w}}=0, v=0.3, \delta=0.1, \delta_{\mathrm{w}}=0.04$. 


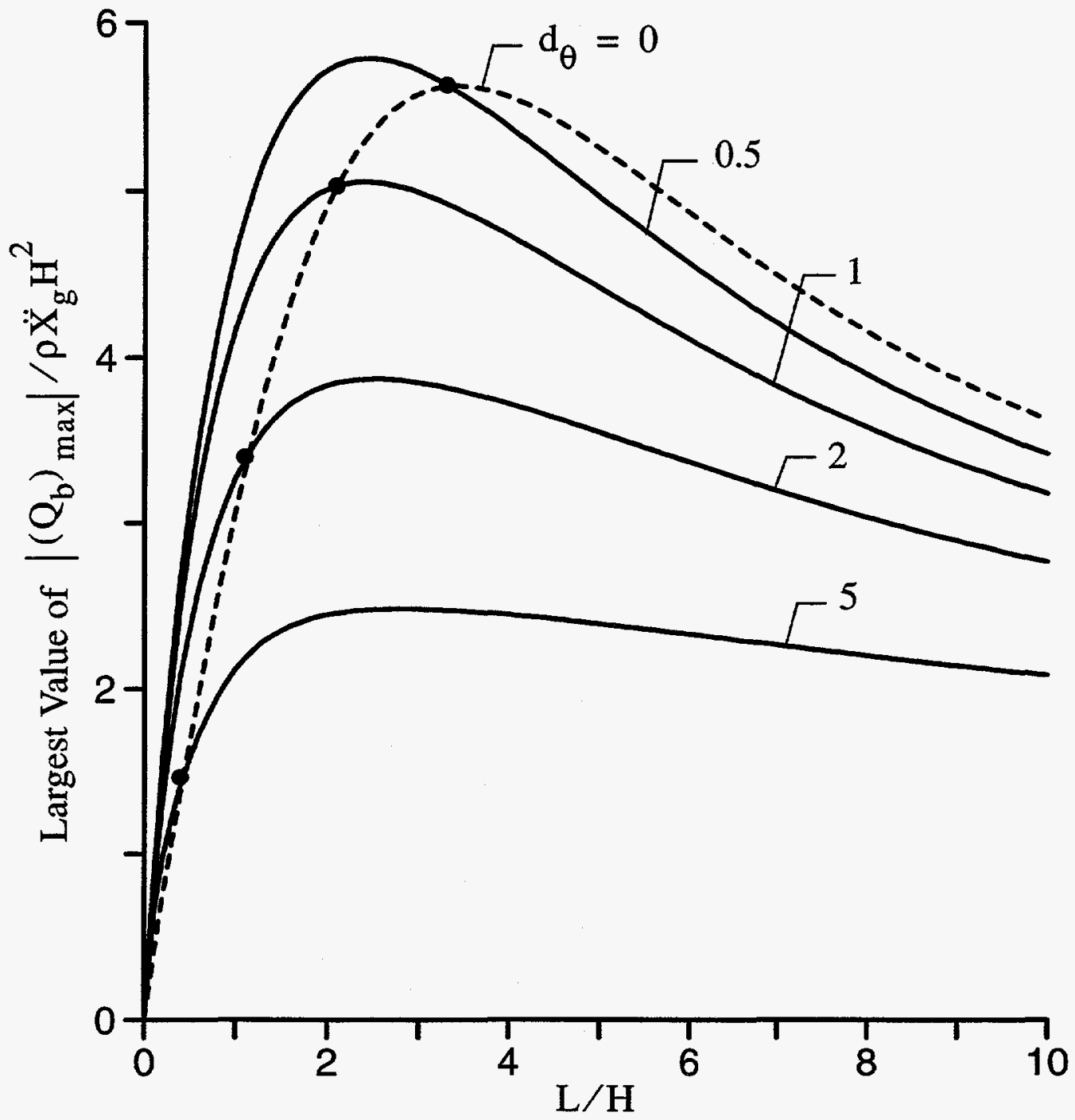

Figure 4.5 Absolute maximum value of base shear per unit of length of each wall for harmonically excited systems with elastically constrained walls; $\mathrm{m}_{\mathrm{w}}=0, \mathrm{v}=0.3, \delta=0.1, \delta_{\mathrm{w}}=0.04$. 


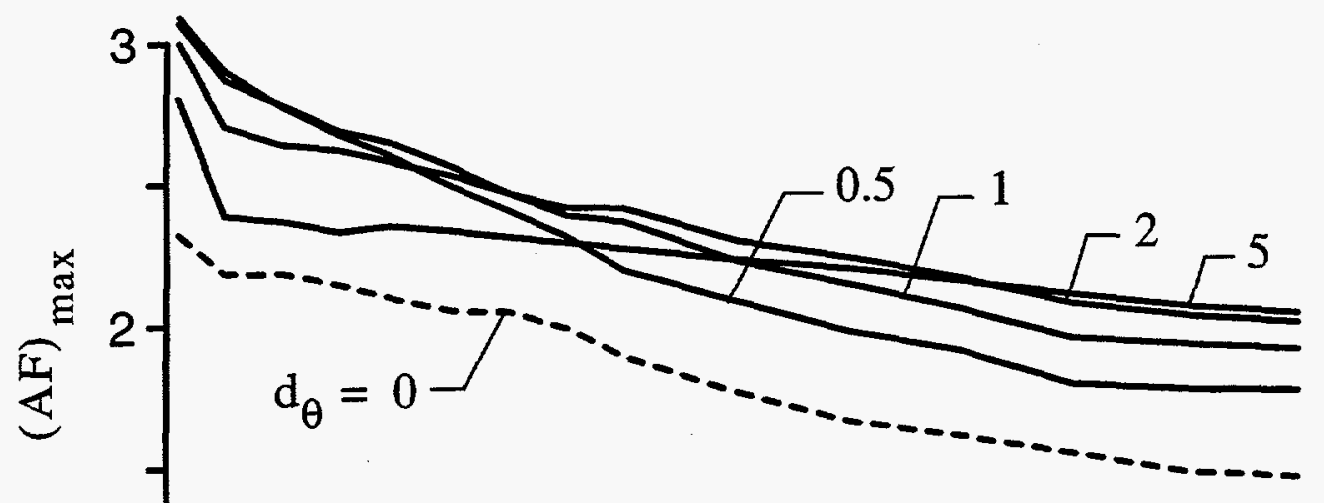

(a) For El Centro Input

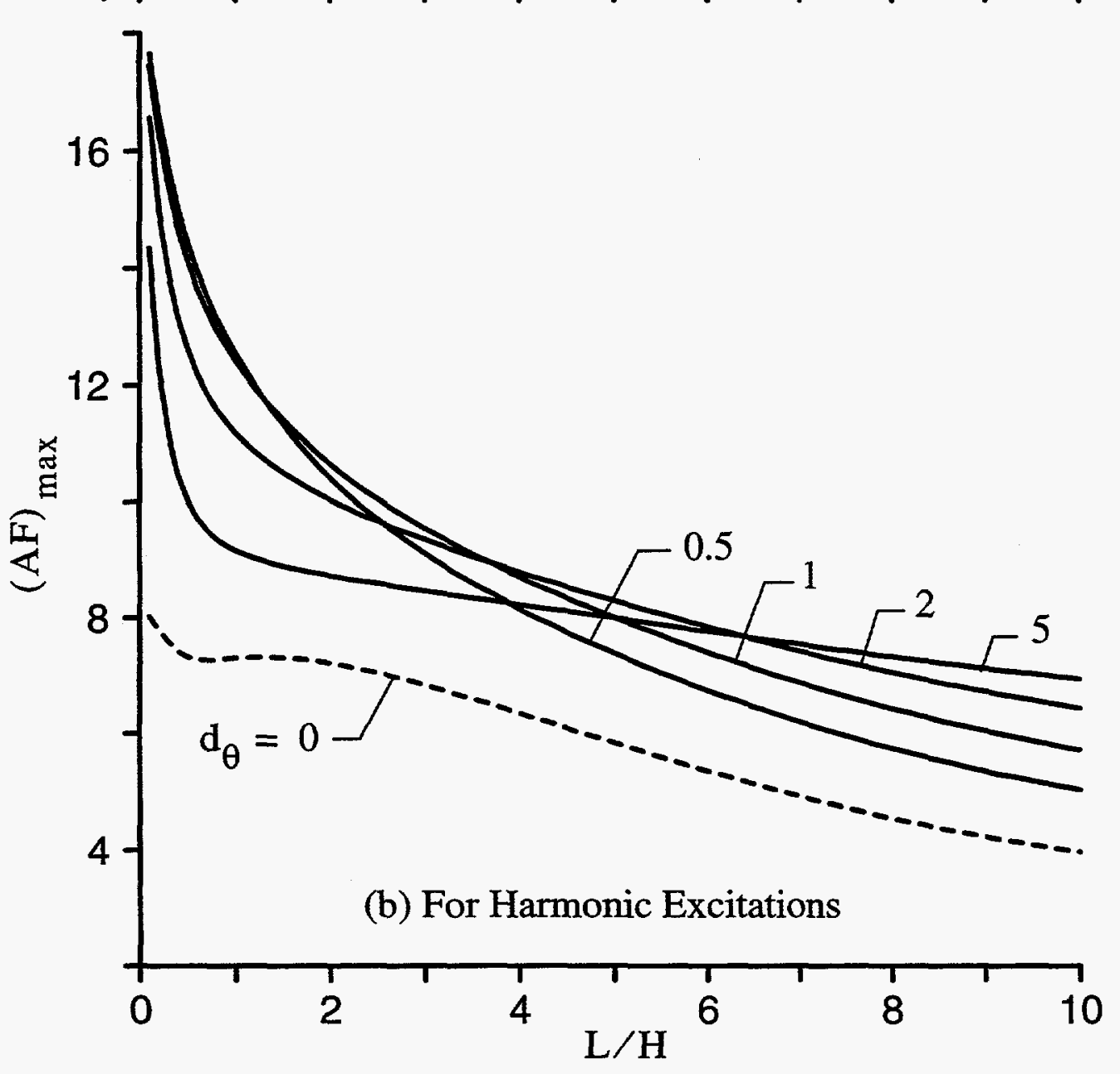

Figure 4.6 Absolute maximum amplification factor for base wall-shear for systems with elastically constrained walls subjected to: (a) the El Centro record; and (b) harmonic base motions; $\mathrm{m}_{\mathrm{w}}=0, v=0.3, \delta=0.1, \delta_{\mathrm{w}}=0.04$. 


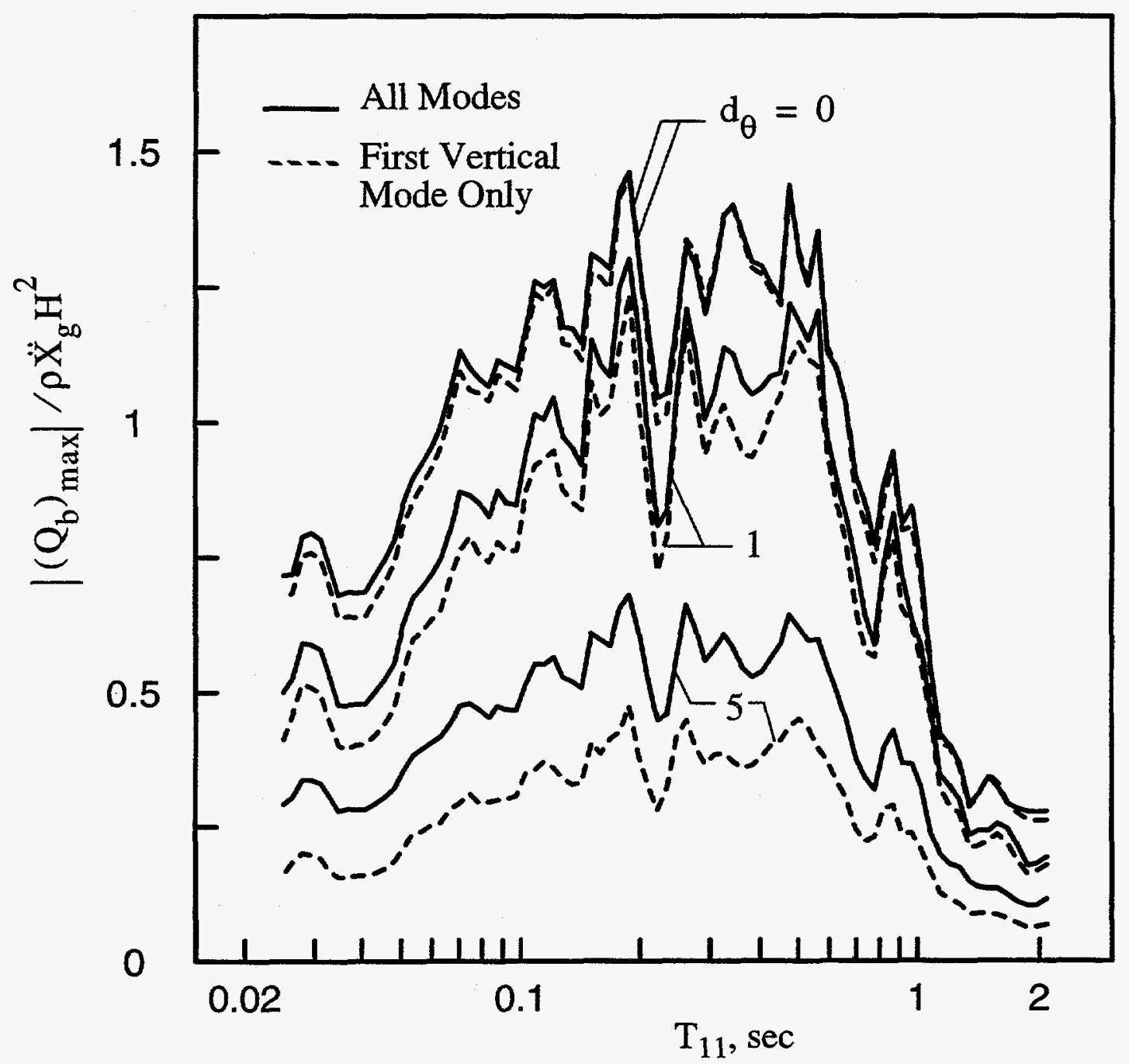

Figure 4.7 Comparison of maximum base wall-shear for systems with elastically constrained walls computed by considering many and a single vertical mode of vibratin; El Centro input, $\mathrm{m}_{\mathrm{w}}=0, v=0.3, \delta=0.1, \delta_{\mathrm{w}}=0.04$. 


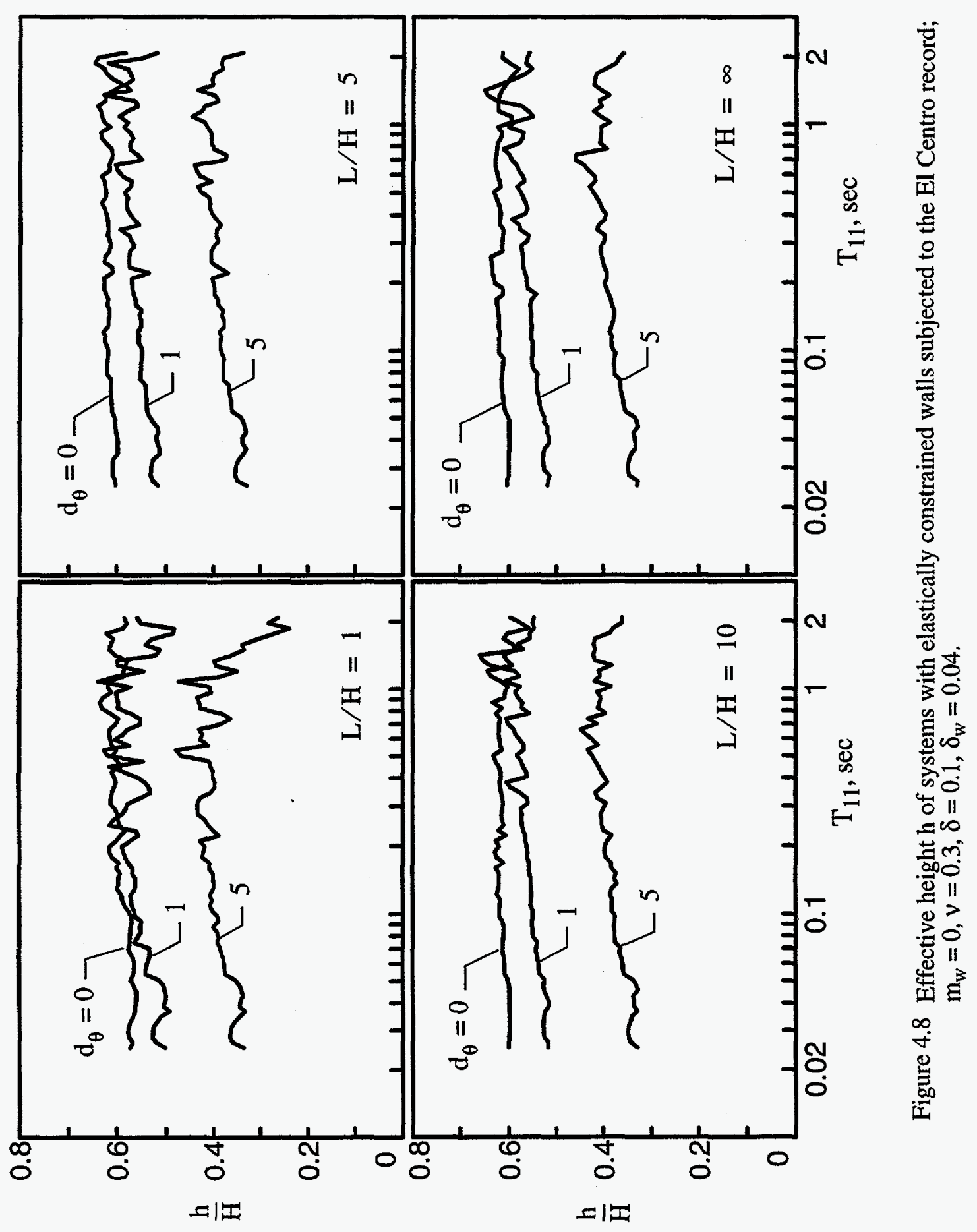




\section{SECTION 5}

\section{CONCLUSIONS}

With the method of analysis presented, the response of the wall-medium system considered may be evaluated simply and with high degree of accuracy. The analysis by this method may be implemented with a small fraction of the effort required by Wood's precise method, and it is free of the numerical instabilities that characterize the latter approach. The accuracy of the method has been demonstrated by comparing its predictions for the special case of fixed-based walls with those obtained by the exact approach. The comprehensive numerical data that have been presented provide valuable insights into the effects and relative importance of the various parameters, and a sound framework for the planning, implementation, and interpretation of the results of analyses for much more complex systems as well. The principal conclusions of this study may be summarized as follows:

1. Almost independently of the characteristics of the ground motion, the magnitudes of the wall pressures and of the associated wall forces induced by ground shaking are highly dependent on the ratio $\mathrm{L} / \mathrm{H}$ of the distance between walls to the height of the retained material. For very slender systems with small values of $\mathrm{L} / \mathrm{H}$, the inertia forces for all the contained material are effectively transmitted to the walls by horizontal extensional action, and practically the entire mass of the medium may be considered to be effective. With increasing $\mathrm{L} / \mathrm{H}$, a progressively larger portion of the inertia forces gets transferred by horizontal shearing action to the base, and the portion of the retained mass that contributes to the wall forces is reduced. Additionally, an increase in $\mathrm{L} / \mathrm{H}$ increases the damping capacity of the system and reduces the dynamic amplification factors for the wall forces.

2. Provided the aspect ratio of the system $\mathrm{L} / \mathrm{H}$ is not very small, the effect of wall flexibility is to reduce the horizontal extensional stiffness of the contained material relative to its shearing stiffness, and this reduction, in turn, reduces the magnitudes of the resulting wall forces. This result is in contradiction to the well established behavior of liquid-containing tanks, for which the effect of wall flexibility is to increase rather than decrease the dominating, impulsive components of response. However, as L/H tends to zero, the medium's ability to transfer forces vertically by horizontal shearing action vanishes, the medium effectively acts as a liquid, and, in conformance with the known response of liquid containing tanks, the flexibility of the wall increases the dynamic effects. For the range of parameters likely to be encountered in practice, the dynamic wall forces for tanks containing a viscoelastic solid are likely to decrease rather than increase with increasing wall flexibility. 
3. While the low-frequency, static values of the wall forces decrease with decreasing distance between walls, the maximum dynamic forces first increase and then decrease. The increased dynamic effects are due to the reduced damping capacity of the finite-sized, narrower systems.

4. For systems with fixed-based rigid walls, an excellent approximation to the response may be obtained by considering the contribution of only the fundamental vertical mode of vibration. By contrast, for systems with elastically constrained walls, the contributions of the second and higher vertical modes may also be important. The contributions of all horizontal modes of vibration are provided for directly in the method of analysis employed.

5. Because of the assumption of vanishing vertical normal stresses underlying the simplified method of analysis, the component of the foundation moment contributed by the dynamic pressures acting on the tank base cannot be evaluated. However, the total foundation moment may be determined directly from the inertia forces of the retained medium, and, if desired, the component contributed by the base pressures may be determined by substracting from the total moment the moments at the base of the walls. 


\section{SECTION 6}

\section{REFERENCES}

1. J. H. Wood, 'Earthquake-induced soil pressures on structures', Report EERL 73-05, Earthquake Engineering Research Laboratory, California Institute of Technology, CA., 1973.

2. J. H. Wood, 'Earthquake-induced pressures on rigid wall structure', Bull. of New Zealand soc. for earthquake eng. 8(3), 175-186, (1975).

3. H. Matuo and S. Ohara, 'Lateral earth pressure and stability of quay walls during earthquakes', Proc. 2nd world conf. earthquake eng. Tokyo, Japan, (1960).

4. A. Arias, F. J. Sanchez-Sesma and E. Ovando-Shelley, 'A simplified elastic model for seismic analysis of earth-retaining structures with limited displacements', Proc. Int. conf. on recent advances in geotech. earthquake eng. and soil dyn., University of Missouri, Rolla, Mo., I, 235-240, 1981.

5. A. S. Veletsos, and A. H. Younan, 'Dynamic soil pressures on rigid vertical walls', Earthquake eng. struct. dyn. 23(3), 275-301, (1994); also available as Report 52357, Brookhaven National Laboratory, Upton, N.Y.

6. A. S. Veletsos, and A. H. Younan, 'Dynamic modeling and response of soil-wall systems', J. geotech. eng., ASCE 120(12), 2155-2179, (1994); also available as Report 52402, Brookhaven National Laboratory, Upton, N.Y.

7. A. S. Veletsos and B. Verbic, 'Vibration of viscoelastic foundations', Earthquake eng. struct. dyn. 2(1), 87-102, (1973).

8. A. S. Veletsos and V. V. D. Nair, 'Seismic Interaction of structures on hysteretic foundations', $J$. struct. div. ASCE 101(1), 109-129, (1975).

9. A. S. Veletsos and K. W. Dotson, 'Horizontal impedances for radially inhomogeneous viscoelastic soil layers', Earthquake eng. struct. dyn. 16(7), 947-966, (1988).

10. J. M. Roesset, R. V. Whitman and R. Dobry, 'Modal analysis for structures with foundation interaction', J. struct. div. ASCE 99(3), 399-416, (1973).

11. J. L. Tassoulas and E. Kausel, 'On the effects of the rigid sidewall on the dynamic stiffness of embedded circular footings', Earthquake eng. struct. dyn. 11(3), 403-414, (1983). 
12. A. Pais and E. Kausel, 'Approximate formulas for dynamic stiffnesses of rigid foundations', Soil dyn. earthquake eng. 7(4), 213-227, (1988).

13. A. S. Veletsos and Y. Tang, 'Deterministic assessment of effects of ground-motion incoherence', J. eng. mech. ASCE 116(5), 1109-1124, (1990).

14. A. S. Veletsos, 'Seismic response and design of liquid storage tanks,' Guidelines for the Seismic Design of Oil and Gas Pipeline Systems, Technical Council on Lifeline Earthquake Engineering, ASCE, New York, 255-370 and 443-461, 1984. 


\section{SECTION 7}

\section{NOTATION}

The following symbols are used in this study:

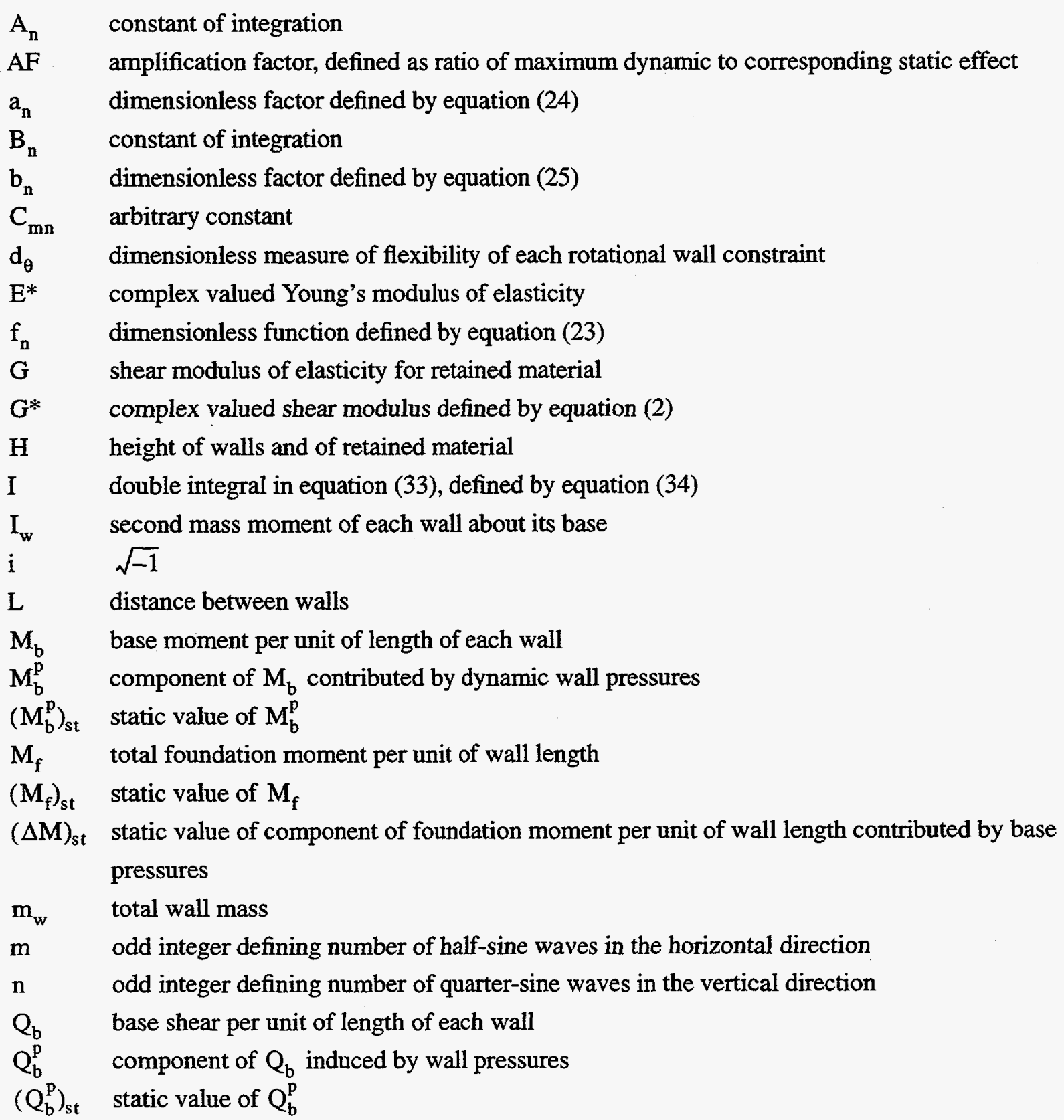




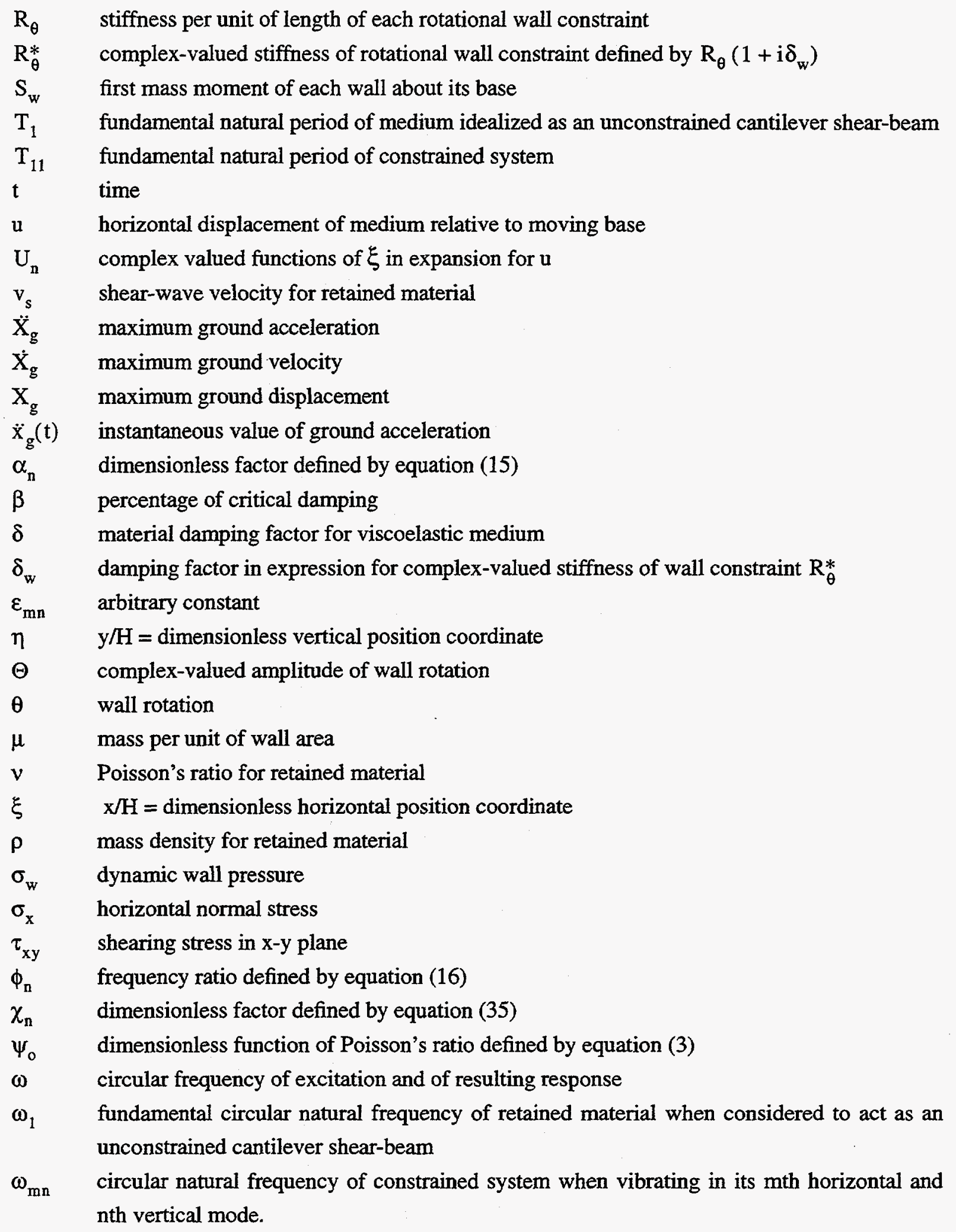

